\title{
PRIME REGULAR HOPF ALGEBRAS OF GK-DIMENSION ONE
}

\author{
K.A. BROWN AND J.J. ZHANG
}

\begin{abstract}
This paper constitutes the first part of a program to classify all affine prime regular Hopf algebras $H$ of Gelfand-Kirillov dimension one over an algebraically closed field of characteristic zero. We prove a number of properties of such an algebra, list some classes of examples, and then prove that - when the PI-degree of $H$ is prime - our list contains all such algebras.
\end{abstract}

\section{INTRODUCTION}

0.1. Recent years have seen substantial progress in our understanding of (infinite dimensional) noetherian Hopf algebras [BG1, LWZ, WZ1, BZ. One noteworthy aspect of this development has been the increasing role of homological algebra - for example, it was shown in WZ1 that every affine noetherian Hopf algebra satisfying a polynomial identity is Auslander-Gorenstein and Cohen-Macaulay, and hence, by [BZ, Lemma 6.1], is Artin-Schelter (AS) Gorenstein; see (1.2) for the definition of the latter condition.

Recall that, by fundamental results of Small, Stafford and Warfield [SSW], a semiprime affine algebra of GK-dimension one is a finite module over its center, which is affine and hence noetherian. Given the homological developments mentioned above, it seems to us a feasible target to determine all prime affine Hopf algebras of GK-dimension one. In this paper we begin the campaign by considering the case where

$H$ is an affine noetherian Hopf algebra over an algebraically closed field $k$ of characteristic 0 , and $H$ has GK-dimension one and is prime and regular.

Throughout, we'll say that $H$ satisfies hypotheses $(\mathbf{H})$ when the displayed conditions are in force. Here, regular means that $H$ has finite global dimension, say $d$. In fact the Cohen-Macaulay property of $H$ forces $d=1$ here, so the objects of study are hereditary noetherian prime rings.

0.2. Examples. By a basic result in the theory of algebraic groups $\mathrm{Hu}$, Theorem 20.5], there are two commutative Hopf $k$-algebras which are domains of GKdimension one, namely $k[x]$ and $k\left[x^{ \pm 1}\right]$, the coordinate rings of $(k,+)$ and $(k \backslash\{0\}, \times)$ respectively. Since these groups are abelian, their coordinate rings are cocommutative; if one retains the cocommutativity hypothesis, but drops commutativity, then the only additional algebras satisfying $(\mathbf{H})$ which enter the arena are the group algebras $k \mathbb{D}$ of the infinite dihedral group (3.2), and the algebras which we call here the cocommutative Taft algebras. These, discussed in (3.3), are skew group algebras

2000 Mathematics Subject Classification. Primary 16E65, 16W30, 16P40; Secondary 16S30, 16S34, 16W35, 16R99 .

Key words and phrases. Hopf algebra, Gelfand-Kirillov dimension, Artin-Schelter regular, homological integral. 
of a finite cyclic group $\langle g\rangle$ of order $n$, acting on $k[x]$, with $g x g^{-1}=\xi x$, where $\xi$ is a primitive $n$th root of one in $k$.

For each $n>1$ there is a finite collection of noncocommutative Taft algebras $H(n, t, \xi)$, also defined in (3.3), for $\xi$ a primitive $n$th root of one in $k$. (Here, $k$ can be any field containing a primitive $n$th root of unity.) The algebras $H(n, t, \xi)$ are well known, being factor algebras of a Borel subalgebra of $U_{\xi}(\mathfrak{s l}(2, k))$.

The above are not, however, the only examples of algebras satisfying $(\mathbf{H})$. A further class of examples was discovered by Liu [Liu] in 2006: these are built by letting a skew primitive element $y$ "of finite index of nilpotency $n$ " act on $k\left[x^{ \pm 1}\right]$ by setting $y x=\xi x y$, and forming a "non-split" extension. Liu's algebras are recovered as a special case of a larger class of algebras in (3.4), where we show that his construction can be extended to include Hopf algebras built over the group algebra $k\left(C_{\infty} \times C_{b}\right)$, where $b$ is an arbitrary divisor of $n$.

In all the examples listed in this subsection, the PI-degree of the algebra is $n$, (where, of course, $n=1$ for the commutative algebras and $n=2$ for $k \mathbb{D}$ ).

0.3 . Prime PI-degree. It may be that the only algebras $H$ satisfying $(\mathbf{H})$ are those listed in (0.2), but we cannot prove this at present. However it is a corollary of our main result that

if $H$ satisfies $(\mathbf{H})$ and the PI-degree $n$ of $H$ is prime, then $H$ occurs in (0.2).

To give a more precise statement of our main result and some idea of its proof, we must introduce some homological algebra.

0.4. Integral order and integral minor. A fundamental tool for the study of algebras satisfying $(\mathbf{H})$ is the homological integral $\int_{H}^{l}$ defined in [LWZ]. This is the one-dimensional $k$-vector space and $H$-bimodule $\operatorname{Ext}_{H}^{1}(k, H)$, where $k$ here denotes the trivial left $H$-module. Let $\pi: H \rightarrow k$ be the algebra map given by the right structure of $\int_{H}^{l}$. As we recall in (2.1), $\pi$ determines groups $G_{\pi}^{l}$ and $G_{\pi}^{r}$ of left and right winding automorphisms of $H$. In our setting, and indeed for any affine Hopf algebra which is a finite module over its center,

$$
n:=\left|G_{\pi}^{l}\right|=\left|G_{\pi}^{r}\right|<\infty,
$$

(Theorem 2.3(c)); the integer $n$ is called the integral order of $H$, denoted $i o(H)$.

When $H$ is commutative, $\pi$ is - clearly - the counit $\epsilon$, and so $i o(H)=1$. So, speaking crudely, $i o(H)$ is a measure of the noncommutativity of $H$; and indeed it will become apparent from our results that, for $H$ satisfying $(\mathbf{H}), i o(H)=1$ only if $H$ is commutative. Notice also that, when $H$ is cocommutative, $G_{\pi}^{l}=G_{\pi}^{r}$; so we can encode the "degree of cocommutativity" of $H$ by means of the index

$$
i m(H):=\left|G_{\pi}^{l}: G_{\pi}^{l} \cap G_{\pi}^{r}\right|
$$

which we call the integral minor of $H$, (2.4). Thus, when $H$ is cocommutative $i m(H)=1$, and, paralleling the corresponding statement for $i o(H)$, it will follow from results in $\S 4$ that, when $H$ satisfies $(\mathbf{H}), \operatorname{im}(H)=1$ only if $H$ is cocommutative.

The connection between the integral order and noncommutativity is in fact more far-reaching than indicated above. By results of LWZ recalled in $\S 2$, for any prime regular affine PI Hopf $k$-algebra, the integral order is a lower bound for the PIdegree; and

when $H$ satisfies $(\mathbf{H}), \quad i o(H)=$ PI- $\operatorname{deg}(H)$. 
In other words, for algebras $H$ satisfying $(\mathbf{H})$ "the non-commutativity of the algebra is entirely encoded by the integral $\int_{H}^{l}$."

0.5. Main theorem. We can now state the main result of this paper:

Theorem. Let $H$ be a Hopf algebra satisfying $(\mathbf{H})$, and suppose that the integral minor im $(H)$ is either 1 or io $(H)$. Then $H$ is one of the algebras listed in (0.2).

In view of (0.4.2) and (0.4.1), the corollary stated in (0.3) follows.

It's worth noting that all the algebras appearing in (0.2) are pointed. We do not know whether this is true for all prime affine noetherian Hopf $k$-algebras of GK-dimension one, nor whether there is a direct way to prove pointedness, without proceeding first to list all the algebras.

The first steps in the proof of the theorem are valid in a much more general setting: we show in $\S 2$, building mainly on work from LWZ, that if a Hopf algebra $A$ has finite integral order $n$, where $n$ is a unit in $k$, then $A$ is strongly graded by the cyclic group of order $n$, in two different ways, with identity components the invariant algebras $A^{G_{\pi}^{l}}$ and $A^{G_{\pi}^{r}}$. When $A$ is in addition regular affine semiprime and has GK-dimension one, these two invariant algebras, and their intersection, are affine Dedekind domains (Theorem 2.5).

$\S 4$ covers the easy case of the main theorem, namely the case where the Hopf algebra $H$ satisfies $(\mathbf{H})$ and $i m(H)=1$. Under these hypotheses, $H_{0}:=H^{G_{\pi}^{l}}=$ $H^{G_{\pi}^{r}}$ and this Dedekind domain is a Hopf subalgebra of $H$. At this point a pivotal dichotomy comes into play: $H_{0}$ must either be $k[x]$ - the primitive case; or $H_{0}$ is $k\left[x^{ \pm 1}\right]$ - the group-like case. When $i m(H)=1$ it's not hard to show that in the primitive case $H$ is a cocommutative Taft algebra, and in the group-like case it's the group algebra of the dihedral group.

The proof of the case $i m(H)=i o(H)$ of the main theorem occupies $\S 5$ and $\S 6$. In $\S 5$ an analysis of algebras strongly graded by a finite abelian group, with commutative component of degree 0 , culminates in the conclusion that, if $H$ satisfies $(\mathbf{H})$ and $i o(H)=i m(H)$, then the Dedekind domain $H_{0}:=H^{G_{\pi}^{l}} \cap H^{G_{\pi}^{r}}$ is the center of $H$ and is a Hopf subalgebra, whence it is either $k[x]$ or $k\left[x^{ \pm 1}\right]$. The dichotomy mentioned in the previous paragraph therefore features again, and in $\S 6$ this is exploited to show that only the algebras of $\S 3$ can occur. This is achieved by first studying the finite dimensional Hopf algebra $H /\left(\operatorname{ker} \epsilon \cap H_{0}\right) H$, which we call the twistor of $H$, and then lifting its structure to describe $H$ itself.

Finally, in $\S 7$, we discuss a number of questions and conjectures arising from this work.

\section{General preparations}

1.1. Standard notation. Throughout, $k$ will denote an algebraically closed field. Unless stated otherwise all vector spaces are over $k$, and an algebra or a ring $A$ always means a $k$-algebra with associative multiplication $m_{A}$ and with unit 1 . All $A$-modules will be by default left modules. Let $A^{\text {op }}$ denote the opposite algebra of $A$.

When $A$ is a Hopf algebra we shall use the symbols $\Delta, \epsilon$ and $S$ respectively for its coproduct, counit and antipode. The coproduct of $a \in A$ will be denoted by $\Delta(a)=\sum a_{1} \otimes a_{2}$. The dual Hopf algebra of $A$ will be denoted by $A^{\circ}$. For background regarding Hopf algebras, see for example [Mo]. 
1.2. Artin-Schelter condition. While our main interest is in Hopf algebras, the following two key definitions apply more generally to any noetherian augmented $k$ algebra - that is, a noetherian $k$-algebra $A$ with a fixed augmentation $\epsilon: A \longrightarrow k$.

Definition. Let $(A, \epsilon)$ be an augmented noetherian algebra.

(a) We shall say $A$ has finite injective dimension if the injective dimensions of ${ }_{A} A$ and $A_{A}, \operatorname{injdim}_{A} A$ and injdim $A_{A}$, are both finite. In this case these integers are equal by [Za], and we write $d$ for the common value. We say $A$ is regular if it has finite global $\operatorname{dimension,~}_{\operatorname{gldim}} A<\infty$. Right global dimension always equals left global dimension [We, Exercise 4.1.1]; and, when finite, the global dimension equals the injective dimension.

(b) Write $k$ for the trivial $A$-module $A / \operatorname{ker} \epsilon$. Then $A$ is Artin-Schelter Gorenstein, which we usually abbreviate to $A S$-Gorenstein, if

(AS1) $\operatorname{injdim}_{A} A=d<\infty$,

(AS2) $\operatorname{dim}_{k} \operatorname{Ext}_{A}^{d}\left({ }_{A} k,{ }_{A} A\right)=1$ and $\operatorname{Ext}_{A}^{i}\left({ }_{A} k,{ }_{A} A\right)=0$ for all $i \neq d$,

(AS3) the right $A$-module versions of (AS1,AS2) hold.

(c) If, further, gldim $A=d$, then $A$ is called Artin-Schelter regular, usually shortened to $A S$-regular.

1.3. Homological integrals. Here is the natural extension to augmented algebras of a definition recently given in [WW] for Hopf algebras. This definition generalises a familiar concept from the case of a finite dimensional Hopf algebra [Mo, Definition 2.1.1]).

Definition. Let $(A, \epsilon)$ be a noetherian augmented algebra, and suppose $A$ is ASGorenstein of injective dimension $d$. Any nonzero element of the one-dimensional $A$-bimodule $\operatorname{Ext}_{A}^{d}\left({ }_{A} k,{ }_{A} A\right)$ is called a left homological integral of $A$. We denote $\operatorname{Ext}_{A}^{d}\left({ }_{A} k,{ }_{A} A\right)$ by $\int_{A}^{l}$. Any nonzero element in $\operatorname{Ext}_{A^{\text {op }}}^{d}\left(k_{A}, A_{A}\right)$ is called a right homological integral of $A$. We write $\int_{A}^{r}=\operatorname{Ext}_{A^{\text {op }}}^{d}\left(k_{A}, A_{A}\right)$. Abusing language slightly, we shall also call $\int_{A}^{l}$ and $\int_{A}^{r}$ the left and the right homological integrals of $A$ respectively. When no confusion as to the algebra in question seems likely, we'll simply write $\int^{l}$ and $\int^{r}$ respectively.

\section{Classical Components}

Although our primary goal is to classify affine prime regular Hopf $k$-algebras of GK-dimension one, we give in this section some preliminary results in a more general setting than is strictly needed for this classification, in the hope that some of these ideas will prove useful in future work. Thus, throughout $₫ 2]$ will be an arbitrary Hopf $k$-algebra.

2.1. Hopf subalgebras associated to winding automorphisms. Given an algebra homomorphism $\pi: A \rightarrow k$, we write $\Xi_{\pi}^{l}$ for the left winding automorphism of $A$ associated to $\pi$, namely,

$$
\Xi_{\pi}^{l}(a)=\sum \pi\left(a_{1}\right) a_{2}
$$

for all $a \in A$; and we use $\Xi_{\pi}^{r}$ for the right winding automorphism of $A$ associated to $\pi$, defined for $a \in A$ by

$$
\Xi_{\pi}^{r}(a)=\sum a_{1} \pi\left(a_{2}\right) .
$$


Fix now a set $\Pi$ of algebra homomorphisms $\pi$ from $A$ to $k$. Let $G_{\Pi}^{l}$ [resp. $G_{\Pi}^{r}$ ] be the subgroup of $\operatorname{Aut}_{k-\text { alg }}(A)$ generated by $\left\{\Xi_{\pi}^{l}: \pi \in \Pi\right\}$ [resp. $\left\{\Xi_{\pi}^{r}: \pi \in \Pi\right\}$ ]. Set $G_{\Pi}:=\left\langle G_{\Pi}^{l}, G_{\Pi}^{r}\right\rangle \subseteq \operatorname{Aut}_{k-\operatorname{alg}}(A)$. (In applications, $A$ will be AS-Gorenstein and we will take $\Pi=\{\pi\}$ with $\pi$ the map $A \rightarrow A / \mathrm{r} \cdot \operatorname{ann}\left(\int^{l}\right)$.)

Let $A_{\Pi}$ [resp. $\left.A_{\Pi}^{l}, A_{\Pi}^{r}\right]$ be the subalgebra of invariants $A^{G_{\Pi}}$ [resp. $\left.A^{G_{\Pi}^{l}}, A^{G_{\Pi}^{r}}\right]$. When $\Pi$ consists of a single map, $\Pi=\{\pi\}$, we'll write $A_{\pi}, A_{\pi}^{l}, A_{\pi}^{r}$, and $G_{\pi}, G_{\pi}^{l}$, $G_{\pi}^{r}$, for these subalgebras of $A$ and subgroups of $\operatorname{Aut}_{k-\text { alg }}(A)$.

The action of $G_{\Pi}^{l}$ is the restriction to the group-like elements $\langle\Pi\rangle \subseteq A^{\circ}$ of the right hit action of $A^{\circ}$ on $A$; and correspondingly for $G_{\Pi}^{r}$ (see e.g. [Mo, 1.6.5 and 1.6.6]).

Proposition. Retain the above notation, and let $\pi, \pi^{\prime} \in \Pi$.

(a) $A_{\Pi}^{l}, A_{\Pi}^{r}$ and $A_{\Pi}$ are subalgebras of $A$ and $A_{\Pi}=A_{\Pi}^{l} \cap A_{\Pi}^{r}$.

(b) If $A$ is cocommutative, then $A_{\Pi}^{l}=A_{\Pi}^{r}=A_{\Pi}$.

(c) $\left(\Xi_{\pi}^{l} \otimes I d_{A}\right) \Delta=\Delta \Xi_{\pi}^{l}$ and $\left(I d_{A} \otimes \Xi_{\pi}^{r}\right) \Delta=\Delta \Xi_{\pi}^{r}$. Thus $A_{\Pi}^{l}$ is a right coideal subalgebra of $A$ and $A_{\Pi}^{r}$ is a left coideal subalgebra of $A$.

(d) $\Xi_{\pi}^{l} \Xi_{\pi^{\prime}}^{r}=\Xi_{\pi^{\prime}}^{r} \Xi_{\pi}^{l}$. In particular, $G_{\Pi}$ is abelian if and only if $G_{\Pi}^{l}$ and $G_{\Pi}^{r}$ are abelian.

(e) $\Xi_{\pi}^{r} S=S\left(\Xi_{\pi}^{l}\right)^{-1}$. Consequently, $S\left(A_{\Pi}^{l}\right) \subseteq A_{\Pi}^{r}$ and $S\left(A_{\Pi}^{r}\right) \subseteq A_{\Pi}^{l}$.

(f) $\left(I d_{A} \otimes \Xi_{\pi}^{l}\right) \Delta=\left(\Xi_{\pi}^{r} \otimes I d_{A}\right) \Delta$. Thus $\Delta(a) \in A \otimes A_{\Pi}^{l}$ if and only if $\Delta(a) \in$ $A_{\Pi}^{r} \otimes A$ if and only if $\Delta(a) \in A_{\Pi}^{r} \otimes A_{\Pi}^{l}$.

(g) Let $\widetilde{A_{\Pi}}:=\left\{a \in A \mid \Delta(a) \in A_{\Pi} \otimes A_{\Pi}\right\}$. Then $\widetilde{A_{\Pi}}$ is also equal to

$$
\begin{aligned}
& \left\{a \in A \mid \Delta(a) \in A_{\Pi}^{l} \otimes A_{\Pi}^{l}\right\}, \\
& \left\{a \in A \mid \Delta(a) \in A_{\Pi}^{r} \otimes A_{\Pi}^{r}\right\},
\end{aligned}
$$

and

$$
\left\{a \in A \mid \Delta(a) \in A_{\Pi}^{r} \otimes A_{\Pi}^{l}\right\} .
$$

(h) $\widetilde{A_{\Pi}}$ is a Hopf subalgebra of $A$. In fact, $\widetilde{A_{\Pi}}$ is the largest Hopf subalgebra of $A_{\Pi}^{l}$, of $A_{\Pi}^{r}$ and of $A_{\Pi}$. If $A_{\Pi}^{l}=A_{\Pi}^{r}$ (and so in particular if $A$ is cocommutative), then $\widetilde{A_{\Pi}}=A_{\Pi}$.

Proof. (a,b) Clear.

(c) Let $\mu$ denote the multiplication map $A \otimes A \longrightarrow A$. Since $\Xi_{\pi}^{l}=\mu\left(\pi \otimes I d_{A}\right) \Delta$,

$$
\begin{aligned}
\left(\Xi_{\pi}^{l} \otimes I d_{A}\right) \Delta & =\left(\mu \otimes I d_{A}\right)\left(\pi \otimes I d_{A} \otimes I d_{A}\right)\left(\Delta \otimes I d_{A}\right) \Delta \\
& =\left(\mu \otimes I d_{A}\right)\left(\pi \otimes I d_{A} \otimes I d_{A}\right)\left(I d_{A} \otimes \Delta\right) \Delta \\
& =\left(\mu \otimes I d_{A}\right)\left(I d_{k} \otimes \Delta\right)\left(\pi \otimes I d_{A}\right) \Delta \\
& =\Delta \Xi_{\pi}^{l} .
\end{aligned}
$$

Similarly, $\left(I d_{A} \otimes \Xi_{\pi}^{r}\right) \Delta=\Delta \Xi_{\pi}^{r}$. Let $a \in A_{\Pi}^{l}$. Then, for all $\pi \in \Pi, \Xi_{\pi}^{l}(a)=a$, and hence

$$
\left(\Xi_{\pi}^{l} \otimes I d_{A}\right) \Delta(a)=\Delta \Xi_{\pi}^{l}(a)=\Delta(a) .
$$

Thus $\Delta(a) \in A_{\Pi}^{l} \otimes A$ and hence $\Delta\left(A_{\Pi}^{l}\right) \subset A_{\Pi}^{l} \otimes A$. Similarly, $\Delta\left(A_{\Pi}^{r}\right) \subset A \otimes A_{\Pi}^{r}$.

(d) Clear.

(e) Note that $\left(\Xi_{\pi}^{l}\right)^{-1}(a)=\sum \pi S\left(a_{1}\right) a_{2}$ for all $a \in A$. Hence, using [Mo, 1.5.10],

$$
\Xi_{\pi}^{r} S(a)=\sum S\left(a_{2}\right) \pi S\left(a_{1}\right)=S\left(\sum \pi S\left(a_{1}\right) a_{2}\right)=S\left(\Xi_{\pi}^{l}\right)^{-1}(a) .
$$

Thus $S\left(A_{\Pi}^{l}\right) \subseteq A_{\Pi}^{r}$. Similarly, $S\left(A_{\Pi}^{r}\right) \subseteq A_{\Pi}^{l}$. 
(f) For $a \in A$,

$$
\left(I d_{A} \otimes \Xi_{\pi}^{l}\right) \Delta(a)=\sum a_{1} \otimes \pi\left(a_{2}\right) a_{3}=\sum a_{1} \pi\left(a_{2}\right) \otimes a_{3}==\left(\Xi_{\pi}^{r} \otimes I d_{A}\right) \Delta(a),
$$

proving the first statement. Now $\Delta(a) \in A \otimes A_{\Pi}^{l}$ if and only if $\left(I d_{A} \otimes \Xi_{\pi}^{l}\right) \Delta(a)=$ $\Delta(a)$ for all $\pi \in \Pi$. By the first assertion, this happens if and only if $\left(\Xi_{\pi}^{r} \otimes\right.$ $\left.I d_{A}\right) \Delta(a)=\Delta(a)$, or, equivalently, $\Delta(a) \in A_{\Pi}^{r} \otimes A$. Using the elementary remark that, for subspaces $A$ and $B$ of a vector space $C$, the intersection of $A \otimes C$ and $C \otimes B$ in $C \otimes C$ is $A \otimes B$ to combine these equivalent statements, one sees that they occur precisely when $\Delta(a) \in A_{\Pi}^{r} \otimes A_{\Pi}^{l}$.

(g) Let $B_{1}=\left\{a \in A \mid \Delta(a) \in A_{\Pi}^{l} \otimes A_{\Pi}^{l}\right\}, B_{2}=\left\{a \in A \mid \Delta(a) \in A_{\Pi}^{r} \otimes A_{\Pi}^{r}\right\}$ and $B_{3}=\left\{a \in A \mid \Delta(a) \in A_{\Pi}^{r} \otimes A_{\Pi}^{l}\right\}$.

Clearly $\widetilde{A_{\Pi}}$ is a subset of $B_{i}$ for $i=1,2,3$. By (f), $B_{1}$ and $B_{2}$ are subsets of $B_{3}$. It remains to show that $B_{3} \subset \widetilde{A_{\Pi}}$.

Suppose that $a \in B_{3}$. Then $\Delta(a)=\sum a_{1} \otimes a_{2}$ for some $a_{1} \in A_{\Pi}^{r}, a_{2} \in A_{\Pi}^{l}$. Consequently,

$$
a=\sum \epsilon\left(a_{1}\right) a_{2}=\sum a_{1} \epsilon\left(a_{2}\right) \in A_{\Pi}^{r} \cap A_{\Pi}^{l}=A_{\Pi} .
$$

By (c),

Thus $a \in \widetilde{A_{\Pi}}$.

$$
\Delta(a) \in\left(A \otimes A_{\Pi}^{r}\right) \cap\left(A_{\Pi}^{l} \otimes A\right) \cap\left(A_{\Pi}^{r} \otimes A_{\Pi}^{l}\right)=A_{\Pi} \otimes A_{\Pi} .
$$

(h) If $b \in \widetilde{A_{\Pi}}$ then $b \in A_{\Pi}$ by (2.1.1).

Since $A_{\Pi}$ is a subalgebra of $A, \widetilde{A_{\Pi}}$ is also a subalgebra of $A$. Since $S\left(A_{\Pi}\right) \subseteq A_{\Pi}$, $S\left(\widetilde{A_{\Pi}}\right) \subseteq \widetilde{A_{\Pi}}$. To prove $\widetilde{A_{\Pi}}$ is a Hopf subalgebra it remains to show that $\widetilde{A_{\Pi}}$ is a subcoalgebra.

Let $a \in \widetilde{A_{\Pi}}$, so that $\Delta(a)=\sum a_{1} \otimes a_{2} \in A_{\Pi} \otimes A_{\Pi}$. Since $\Delta\left(A_{\Pi}\right) \subset A_{\Pi}^{l} \otimes A_{\Pi}^{r}$ and $\left(I d_{A} \otimes \Delta\right) \Delta(a)=\left(\Delta \otimes I d_{A}\right) \Delta(a)$,

$$
\left(I d_{A} \otimes \Delta\right) \Delta(a) \in\left(A_{\Pi}^{l} \otimes A_{\Pi}^{r} \otimes A_{\Pi}\right) \cap\left(A_{\Pi} \otimes A_{\Pi}^{l} \otimes A_{\Pi}^{r}\right)=A_{\Pi} \otimes A_{\Pi} \otimes A_{\Pi} .
$$

Thus $\Delta\left(a_{1}\right), \Delta\left(a_{2}\right) \in A_{\Pi} \otimes A_{\Pi}$. Therefore, $a_{1}, a_{2} \in \widetilde{A_{\Pi}}$ and $\widetilde{A_{\Pi}}$ is a coalgebra.

The claims in the second sentence follow from the first part together with $(\mathrm{g})$.

Suppose finally that $A_{\Pi}^{l}=A_{\Pi}^{r}$. Then by part (c) $A_{\Pi}=A_{\Pi}^{l}=A_{\Pi}^{r}$ is a subcoalgebra of $A$. Also by part (e), $S\left(A_{\Pi}\right) \subseteq A_{\Pi}$. Hence $A_{\Pi}$ is a Hopf subalgebra of $A$. Thus $\widetilde{A_{\Pi}}=A_{\Pi}$.

Write $G\left(A^{\circ}\right)$ for the group of all group-like elements in $A^{\circ}$. So $G\left(A^{\circ}\right)$ is the character group of $A$; that is, by definition, it is $\operatorname{Hom}_{k \text {-alg }}(A, k)$, furnished with the convolution product $*$, BG2, I.9.24]. We record the following obvious facts:

Lemma. Suppose $\langle\Pi\rangle$ is a finite abelian subgroup of $G\left(A^{\circ}\right)$.

(a) $G_{\Pi}^{r}$ and $G_{\Pi}^{l}$ are finite abelian groups, isomorphic to $\langle\Pi\rangle$.

(b) $G_{\Pi}$ is a factor of the direct product of $G_{\Pi}^{l}$ and $G_{\Pi}^{r}$, and in particular is abelian of order at most $\left|G_{\Pi}^{l}\right|^{2}=|\langle\Pi\rangle|^{2}$.

(c) If the order $|\langle\Pi\rangle|$ is coprime to the characteristic $p$ of $k$ when $p>0$, then the character group $\widehat{G_{\Pi}^{l}}$ is isomorphic to $G_{\Pi}^{l}$.

Proof. (a) It is easy to check that the maps from $G\left(A^{\circ}\right)$ to $\operatorname{Aut}_{k-\text { alg }}(A)$ given by $\pi \mapsto \Xi_{\pi}^{r}$ and $\pi \mapsto \Xi_{\pi}^{l}$ are, respectively, a group monomorphism and a group anti-monomorphism. Thus the assertion follows. 
(b) This is immediate from Proposition 2.1(d).

(c) This is standard.

2.2. Strongly graded property. Recall our standing hypotheses for $\S 2$. In addition we assume in this paragraph and for the remainder of this paper that $S$ is bijective; since this holds when $A$ is semiprime or satisfies a polynomial identity $\underline{\mathrm{Sk}}$, it will be the case in the applications we have in mind. Moreover, we assume in this paragraph that

$$
\langle\Pi\rangle \text { is a finite abelian subgroup of } G\left(A^{\circ}\right) \text {, }
$$

and that

$$
|\langle\Pi\rangle| \text { is coprime to the characteristic } p \text { of } k \text {, when } p>0 \text {. }
$$

It will be convenient to write $\widehat{G_{\Pi}^{l}}$ additively. For each $\chi \in \widehat{G_{\Pi}^{l}}$, put

$$
A_{\Pi, \chi}^{l}:=\left\{a \in A \mid g(a)=\chi(g) a, \forall g \in G_{\Pi}^{l}\right\} .
$$

Then $A$ is a $\widehat{G_{\Pi}^{l}}$-graded algebra: $A=\oplus_{\chi \in \widehat{G_{\Pi}^{l}}} A_{\Pi, \chi}^{l}$. In particular $A_{\Pi, 0}^{l}$ consists of the $G_{\Pi}^{l}$-invariants of $A$, which we denoted by $A_{\Pi}^{l}$ in $\$ 2.1$. Similarly, $A$ is $G_{\Pi}^{r}$-graded, with components $A_{\Pi, \chi}^{r}$ for $\chi \in \widehat{G_{\Pi}^{r}}$. The following is well-known:

Lemma. Let $G$ be a finite abelian group acting faithfully and semisimply on an algebra $A$, so $A$ is $\widehat{G}$-graded, $A=\oplus_{\chi \in \widehat{G}} A_{\chi}$. If, for any $A_{\chi} \neq 0$ and $A_{\chi^{\prime}} \neq 0$, $A_{\chi} A_{\chi^{\prime}} \neq 0$ (e.g., $A$ is a $\widehat{G}$-graded domain), then $A_{\chi} \neq 0$ for all $\chi \in \widehat{G}$.

Proof. Let $K=\left\{\chi \in \widehat{G} \mid A_{\chi} \neq 0\right\}$. Since, for any $A_{\chi} \neq 0$ and $A_{\chi^{\prime}} \neq 0, A_{\chi} A_{\chi^{\prime}} \neq 0$, $K$ is a finite subsemigroup of $\widehat{G}$, whence $K$ is a subgroup of $\widehat{G}$. Since $G$ acts faithfully on $A, L:=\{g \in G \mid \chi(g)=1, \forall \chi \in K\}$ is trivial. If $K \neq \widehat{G}$, then $L \neq\{1\}$, yielding a contradiction.

The next result is fundamental to our analysis. We have chosen to give a direct proof, but see Remark (b) below for an alternative approach.

Proposition. Suppose (2.2.1) and (2.2.2).

(a) $\Delta\left(A_{\Pi, \chi}^{l}\right) \subseteq A_{\Pi, \chi}^{l} \otimes A$ for all $\chi \in \widehat{G_{\Pi}^{l}}$.

(b) $A=\oplus_{\chi \in \widehat{G_{\Pi}^{l}}} A_{\Pi, \chi}^{l}$ is strongly $\widehat{G_{\Pi}^{l}}$-graded.

Proof. (a) The assertion is equivalent to the statement that $\left(\Xi_{\pi}^{l} \otimes I d_{A}\right) \Delta(a)=$ $\chi\left(\Xi_{\pi}^{l}\right) \Delta(a)$ for every $a \in A_{\chi}^{l}$, which follows from Proposition 2.1(c).

(b) For this, it suffices to show that $A_{\Pi, \chi}^{l} A_{\Pi,-\chi}^{l}=A_{\Pi, 0}^{l}$ for all $\chi \in \widehat{G_{\Pi}^{l}}$. Since $A$ is $\widehat{G_{\Pi}^{l}}$-graded, we only need to show that $A_{\Pi, \chi}^{l} A$ contains 1 for all $\chi \in \widehat{G_{\Pi}^{l}}$.

Let $\chi \in \widehat{G_{\Pi}^{l}}$ and suppose there is $0 \neq a \in A_{\Pi, \chi}^{l}$. By (a), $\Delta(a)=\sum a_{1} \otimes a_{2}$ with $a_{1} \in A_{\Pi, \chi}^{l}$. Since $a=\sum \epsilon\left(a_{1}\right) a_{2}$, there is a $b \in A_{\Pi, \chi}^{l}$ such that $\epsilon(b) \neq 0$, and after adjusting $a$ we may assume that $\epsilon(b)=1$. Then $\Delta(b)=\sum b_{1} \otimes b_{2}$ with $b_{1} \in A_{\Pi, \chi}^{l}$. Therefore $1=\epsilon(b)=\sum b_{1} S\left(b_{2}\right) \in A_{\Pi, \chi}^{l} A$.

In the above paragraph, we have shown that $A$ is strongly $K$-graded where $K=\left\{\chi \in \widehat{G_{\Pi}^{l}} \mid A_{\Pi, \chi} \neq 0\right\}$. As a consequence, if $A_{\Pi, \chi} \neq 0$ and $A_{\Pi, \chi^{\prime}} \neq 0$, then $A_{\Pi, \chi} A_{\Pi, \chi^{\prime}} \neq 0$. By Lemma 2.1(a), $G_{\Pi}^{l}$ acts faithfully on $A$ and by (2.2.2), $G_{\Pi}^{l}$ acts semisimply on $A$. Lemma 2.2 above says that $K=\widehat{G_{\Pi}^{l}}$. 
Remarks. (a) Of course the right-hand version of the proposition also holds.

(b) In fact the proposition can be deduced from standard results on Hopf-Galois extensions, as follows. Suppose that $\pi: H \rightarrow \bar{H}$ is an epimorphism of Hopf algebras, with $\bar{H}$ finite dimensional. So $H$ is a right $\bar{H}$-comodule algebra via the map $\phi:=(1 \otimes \pi) \circ \Delta: H \rightarrow H \otimes \bar{H}$. Define $B:=H^{c o \bar{H}}=\{b \in H: \phi(b)=b \otimes 1\}$. Then the inclusion of algebras $B \subseteq H$ is $\bar{H}$-Galois by [Sc, Example 3.6] and [Mo, Theorem 8.3.1]. We apply this with $A=H$ and $\bar{H}=\left(k G_{\Pi}^{l}\right)^{*}$ Then $\bar{H}$ is the group algebra $k \widehat{G_{\Pi}^{l}}$ by (2.2.1) and (2.2.2), and so $A$ is strongly $\widehat{G_{\Pi}^{l}}$-graded by Mo, Theorem 8.1.7].

2.3. Classical components. At this point we need to recall and refine some definitions introduced in [LWZ, §7]; at the same time we take the opportunity to simplify our notation.

Definition. Let $A$ be an AS-Gorenstein Hopf algebra with bijective antipode, and let $\pi$ be the algebra homomorphism from $A$ onto $A / \mathrm{r}$. $\operatorname{ann}\left(\int_{A}^{l}\right)$, where $\int_{A}^{l}$ denotes the left integral of Definition 1.3

(a) $A_{\pi}^{l}, A_{\pi}^{r}$ and $A_{\pi}$ are respectively called the left classical component, the right classical component, and the classical component of $A$. These subalgebras of $A$ will respectively be denoted by $A_{0}^{l}, A_{0}^{r}$ and $A_{0}$.

(b) The integral order io $(A)$ of $A$ is the order of $\pi$ in the group $G\left(A^{\circ}\right)$.

(c) The integral annihilator of $A$ is the maximal Hopf ideal (see Remark (c) below) contained in r.ann $\left(\int_{A}^{l}\right)$. It is denoted by $J_{i q}$.

(d) The Hopf algebra $A / J_{i q}$ is called the integral quotient of $A$, denoted by $A_{i q}$.

Remarks A. (a) In [LWZ, §7], $A_{0}^{r}$ is called the classical component of $A$. But, as we'll see in Examples 3.2 and [3.4, $A_{0}^{r}$ is not always a Hopf subalgebra of $A$. We'll provide evidence below in Theorems 4.1 and 5.2 that $A_{0}$ may always be a normal Hopf subalgebra of $A$ when $A$ is prime regular affine of GK-dimension one, so the present definition seems more appropriate.

(b) By Lemma 2.1(a), io $(A)=\left|G_{\pi}^{l}\right|=\left|G_{\pi}^{r}\right|$.

(c) It is easy to see that there is a unique largest Hopf ideal contained in any (two-sided) ideal of a Hopf algebra, so $J_{i q}$ is well-defined. Since the antipode $S$ is bijective, $S\left(\int_{A}^{l}\right)=\int_{A}^{r}$, and so the definitions of the integral annihilator (and thus the integral quotient) are right-left symmetric: $J_{i q}$ is the biggest Hopf ideal in l.ann $\left(\int_{A}^{r}\right)$. In a similar fashion one can check that the various classical components can all equally well be defined using the left annihilator of the right integral.

(d) It will be convenient later (see Hypotheses (2.5)) to extend the use of the notation $A_{0}^{l}, A_{0}^{r}, A_{0}$ in Definition 2.3 (a) to cases where the algebra homomorphism $\pi$ is not necessarily given by the left integral.

Let us now review some basic facts about how the above concepts behave in the case of a Hopf algebra which is a finite module over its center. Recall that, if $Z$ is an Ore domain, then the rank of a $Z$-module $M$ is defined to be the $Q(Z)$-dimension of $Q(Z) \otimes_{Z} M$, where $Q(Z)$ is the quotient division $\operatorname{ring}$ of $Z$; the rank of a right $Z$-module is defined analogously. Let $R$ be any algebra. The PI-degree of $R$ is defined to be

$$
\mathrm{PI}-\operatorname{deg}(R)=\min \left\{n \mid R \hookrightarrow M_{n}(C) \text { for some commutative } \operatorname{ring} C\right\}
$$


(see [MR, Ch. 13]). If $R$ is a prime PI ring with center $Z$, then the PI-degree of $R$ equals the square root of the rank of $R$ over $Z$.

Part (b) below was previously known only under the extra hypothesis that $A$ is regular [LWZ, Lemma 5.3(g)].

Theorem. Let $A$ be an affine Hopf $k$-algebra which is a finite module over its center.

(a) A is noetherian, $A S$-Gorenstein and has a bijective antipode.

(b) The integral order io $(A):=n$ of $A$ is finite.

(c) If $A$ is prime and $Z(A)$ is integrally closed, then io $(A) \leq \mathrm{PI}-\operatorname{deg}(A)$. In particular this holds if $A$ is regular and prime.

(d) The integral quotient $A_{i q}$ is isomorphic as a Hopf algebra to $\left(k C_{n}\right)^{\circ}$, the Hopf dual of the group algebra of the cyclic group of order $n$.

(e) $J_{i q}=\cap_{i=0}^{n-1} \operatorname{ker}\left((\pi)^{i}: A \rightarrow k\right)$.

(f) Suppose that $A$ is regular of global dimension $d$, and that $n$ is a unit in $k$. Then $A_{0}^{l}$ and $A_{0}^{r}$ are both regular of global dimension $d$.

Proof. (a) The Artin-Tate Lemma [MR, 13.9.10] shows that $A$ is noetherian. That $A$ is AS-Gorenstein is a special case of the main result of [WZ1. Bijectivity of the antipode is proved in $\mathrm{Sk}$.

(b) We'll prove that io $(A)$ is bounded above by the number of maximal ideals of $A$ whose intersection with $Z(A)$ equals $\operatorname{ker} \epsilon \cap Z(A)$, which of course is finite because $A$ is a finite $Z(A)$-module.

By the definition of $\int_{A}^{l}, \operatorname{ker} \epsilon \cap Z(A) \subseteq \operatorname{r} \cdot \operatorname{ann}\left(\int_{A}^{l}\right)$. Hence, by Muller's theorem BG2, Theorem III.9.2], $\operatorname{ker} \epsilon$ and r.ann $\left(\int_{A}^{l}\right)$ belong to the same clique of maxspec $A$ (in the sense of [BG2, I.16.2], for example). This means that there is a positive integer $t$ and a set $k=V_{0}, \ldots, V_{t}=\int_{A}^{l}$ of simple right $A$-modules such that, for each $j=0, \ldots, t-1$, either $\operatorname{Ext}_{A}^{1}\left(V_{j+1}, V_{j}\right) \neq 0$ or $\operatorname{Ext}_{A}^{1}\left(V_{j}, V_{j+1}\right) \neq 0$. Now, since $\int_{A}^{l}$ is a one-dimensional (right) $A$-module, it has an inverse in $G\left(A^{\circ}\right)$, given in fact by $\left(S\left(\int_{A}^{l}\right)\right)^{*}$. Hence, the functor $\int_{A}^{l} \otimes-$ is an auto-equivalence of the category of right $A$-modules. In particular $\operatorname{Ext}_{A}^{i}(M, N) \cong \operatorname{Ext}_{A}^{i}\left(\int_{A}^{l} \otimes M, \int_{A}^{l} \otimes N\right)$ for all non-negative integers $i$ and all right $A$-modules $M$ and $N$. Applying this to the above chain of Ext-groups, we deduce that (the annihilators of) $\int_{A}^{l} \otimes k \cong \int_{A}^{l}$ and $\left(\int_{A}^{l}\right)^{\otimes 2}$ are in the same clique. That is, $\left(\int_{A}^{l}\right)^{\otimes 2}$ is in the same clique as $k$. Repeating this argument allows us to deduce that $\left(\int_{A}^{l}\right)^{\otimes m}$ is in the clique of $k$ for all nonnegative integers $m$. Now the finiteness of clique $(\operatorname{ker} \epsilon)$ noted at the start of the argument forces $\left(\int_{A}^{l}\right)^{\otimes r} \cong\left(\int_{A}^{l}\right)^{\otimes s}$ for non-negative integers $r, s$ with $r>s$. Thus

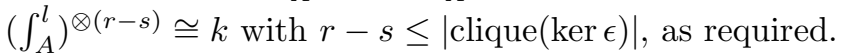

(c) The extra hypotheses ensure that $A$ equals its trace ring, so that

$$
\mid \text { clique(ker } \epsilon) \mid \leq \mathrm{PI}-\operatorname{deg}(A)
$$

by [Bra, Theorem 8$]$. When $A$ is regular its center is integrally closed by [LWZ, Lemma 5.3(b)].

(d),(e) These are special cases of [LWZ, Lemma 4.4(b),(c)].

(f) By the strong grading property, Proposition 2.2(b), $A_{0}^{l}$ is a direct summand of $A$ as left $A_{0}^{l}$-module. So, if $V$ is any left $A_{0}^{l}$-module, then

$$
\operatorname{projdim}_{A_{0}^{l}}(V) \leq \operatorname{projdim}_{A_{0}^{l}}\left(A \otimes_{A_{0}^{l}} V\right) \leq \operatorname{projdim}_{A}\left(A \otimes_{A_{0}^{l}} V\right),
$$


where the second inequality holds because $A$ is a projective left $A_{0}^{l}$-module. Therefore $\operatorname{gldim}\left(A_{0}^{l}\right) \leq d$. However, since $A$ is a finitely generated $A_{0}^{l}$-module, these algebras have the same GK-dimension, and this is $d$ thanks to the Cohen-Macaulay property satisfied by A, BG1, Theorem A]. Since Krull dimension equals GKdimension and is a lower bound for global dimension for noetherian rings which are finite over their centers [GS, the result follows.

Proposition. Let $A$ be an AS-Gorenstein Hopf algebra with a bijective antipode. Assume (2.2.2) for $\Pi=\{\pi\}$, where $\pi: A \rightarrow A / \mathrm{r} \cdot \operatorname{ann}\left(\int_{A}^{l}\right)$. Then

$$
J_{i q}=\left(J_{i q} \cap A_{0}^{l}\right) A=A\left(A_{0}^{l} \cap J_{i q}\right)=\left(\operatorname{ker} \epsilon \cap A_{0}^{l}\right) A .
$$

Proof. By Theorem 2.3(e), $J_{i q}$ is stable under the action of $G_{\pi}^{l}$ on $A$. Thus $J_{i q}$ is $\widehat{G_{\pi}^{l}}$-graded. The first two equalities therefore follow at once from strong grading, Proposition 2.2(b). Moreover, since $J_{i q} \subseteq \operatorname{ker} \epsilon, J_{i q} \subseteq\left(\operatorname{ker} \epsilon \cap A_{0}^{l}\right) A$. But the factors by both these right ideals have $k$-dimension $n$, by Proposition 2.2(b) and Theorem 2.3(d), so equality follows.

Remarks B. (a) The hypothesis (2.2.2) that $p \nmid i o(A)$ is necessary for the validity of Proposition 2.2. For, let $k$ have characteristic $p>0$, and let $A$ be the factor of the enveloping algebra of the $k$-Lie algebra $\mathfrak{g}=\{k x \oplus k y:[y, x]=x\}$ by the Hopf ideal $\left\langle y^{p}-y\right\rangle$. Continue to write $x$ and $y$ for the images of these elements in $A$. Then, by [BZ, Lemma 6.3(c)], $\Xi_{\pi}(x)=x$ and $\Xi_{\pi}(y)=y-1$, so that $G_{\pi}^{l}=G_{\pi}^{r}=\left\langle\Xi_{\pi}\right\rangle \cong C_{p}$, and $A_{0}^{r}=A_{0}^{l}=A_{0}=k[x]$. Thus $A=\bigoplus_{i=0}^{p-1} A_{0} y^{i}$, but this is not a strong group grading. In fact $A$ has no non-trivial $\mathbb{Z}_{p}$-grading that is a strong grading. Observe also that this example is regular (and so hereditary): for by [LL it's enough to check that the trivial $A$-module has finite projective dimension, and this follows since it is a direct summand of the semisimple module $A / A x$, which manifestly has projective dimension one. Notice finally that $J_{i q}=\langle x\rangle$, so that Proposition 2.3 remains valid for this example. We do not know if (2.2.2) is actually needed for Proposition 2.3 or for Theorem 2.3(f).

(b) Given the known examples, we ask:

Question: Under the hypotheses of Proposition 2.3 , is the center $Z(A)$ of $A$ contained in the classical component $A_{0}$ ?

We shall show in Lemma 5.2 that this is the case when $A$ is prime regular of GK-dimension 1, but it may be that it is true much more generally.

(c) Of course the version of Proposition 2.3 with $A_{0}^{r}$ replacing $A_{0}^{l}$ is also true.

(d) In fact (d) and (e) of Theorem 2.3 hold, with the proofs from [LWZ, in any Hopf algebra with finite integral order.

(e) We do not know if all the parts of Theorem 2.3 remain valid if the hypotheses are weakened to include all affine noetherian prime Hopf $k$-algebras which satisfy a polynomial identity. We note here that the question is not vacuous, since not all such algebras are finite modules over their centers. Thus the algebra $\bar{U}$ constructed in GL as the bosonisation of the enveloping algebra of a Lie superalgebra is such an algebra, of GK-dimension 2, which is not a finite module over its center (as can be most quickly confirmed by checking that the clique of the augmentation ideal is infinite).

2.4. The integral minor. In addition to the integral order, our classification of Hopf algebras of GK-dimension one will involve a second invariant, which - crudely speaking - is a measure of the extent of left-right asymmetry between the right and 
left hit actions of $\pi$, the group-like element arising from the left integral. More precisely, it measures the difference between $G_{\pi}$ and $G_{\pi}^{l} \times G_{\pi}^{r}$. The definition is as follows.

Definition. Let $A$ be a Hopf algebra and keep the notation and hypotheses of Definition 2.3. Recall from 2.1 that $G_{\pi}$ is an abelian subgroup of $\operatorname{Aut}_{k-\operatorname{alg}}(A)$ generated by its subgroups $G_{\pi}^{l}$ and $G_{\pi}^{r}$. Suppose that the integral order $i o(A)$ is finite; or, equivalently, that $G_{\pi}$ is a finite group. The integral minor of $A$ is

$$
\operatorname{im}(A):=\left|G_{\pi}^{l} /\left(G_{\pi}^{l} \cap G_{\pi}^{r}\right)\right|,
$$

the intersection being taken as subgroups of $G_{\pi}$.

In particular, $i m(A)=1$ if and only if $G_{\pi}^{l}=G_{\pi}^{r}$, and this holds in particular when $A$ is cocommutative.

2.5. Classical components in GK-dimension one. In preparation for the work on Hopf algebras of GK-dimension one which will occupy the rest of the paper, we state here as our starting point what can immediately be written down about such an algebra using the results in [WW and the concepts introduced in this section.

Theorem. Let $A$ be a semiprime affine Hopf $k$-algebra of GK-dimension one, with $\pi$ the homomorphism from $A$ onto $A / \mathrm{r} \cdot \operatorname{ann}\left(\int_{A}^{l}\right)$.

(a) The integral order io $(A):=n$ of $A$ is finite.

(b) If (2.2.2) holds for $\Pi=\{\pi\}$, then $A$ is strongly graded in two ways by the cyclic group of order $n$, namely

$$
A=\oplus_{\chi \in \widehat{G_{\pi}^{l}}} A_{\pi, \chi}^{l} \text { and } A=\oplus_{\chi \in \widehat{G_{\pi}^{r}}} A_{\pi, \chi}^{r} .
$$

(c) The injective dimension of $A$ is one.

Suppose in addition for (d), (e) and ( $f)$ that $A$ is regular; for $(e)$ and $(f)$, assume also that it is prime, and for $(f)$ assume also (2.2.2).

(d) $A$ is hereditary.

(e) $\mathrm{PI}-\operatorname{deg}(A)=n$.

(f) $A_{0}^{l}, A_{0}^{r}$ and $A_{0}$ are affine commutative Dedekind domains, with $A_{0}^{l} \cong A_{0}^{r}$.

Proof. By [SSW], $A$ is noetherian and is a finite module over its center. Thus $A$ is AS-Gorenstein and Cohen-Macaulay, by WZ1, Theorems 01. and 0.2(1)], so $A$ has a left integral and $\pi$ is defined for $A$.

(a) follows from Theorem 2.3(b). (b) is immediate from Proposition 2.2. (c) follows from WZ1, Theorem 0.2(3)].

Suppose henceforth that $A$ is regular. Then (c) immediately gives (d).

(e) That the PI-degree of $A$ equals its integral order when $A$ is prime and regular is part of [LWZ, Theorem 7.1].

(f) That $A_{0}^{l}$ is an affine commutative domain is proved in [LWZ, Theorem 7.1]; that it is Dedekind now follows from Theorem 2.3(f). An isomorphism of the right and left classical components is provided by the antipode, thanks to Proposition 2.1(e). Finally, $A_{0}=\left(A_{0}^{l}\right)^{G_{\pi}^{r}}=\left(A_{0}^{r}\right)^{G_{\pi}^{l}}$ by Proposition 2.1(d). Thus $A_{0}$ is an affine commutative domain, and since the fixed ring of an integrally closed commutative domain under the action of a finite group is again integrally closed, it follows that $A_{0}$ is Dedekind. 
Remark. The equality in (e) fails when any one of the hypotheses that $A$ is prime, regular, or has GK-dimension one, is dropped. To see the need for the prime hypothesis, one can take $A=\mathbb{C} G$ where $G$ is any direct product of a finite group with an infinite cyclic group. In this case $\int_{A}^{l} \cong k$ and so $i o(A)=1$. The regular hypothesis fails in Case 2 of Example 3.2 in the next section; here, the integral order is 1 but the PI-degree is 2. Finally, (e) fails for prime regular affine PI Hopf algebras of GK-dimension $\geq 2$, see [LWZ, Example 8.5]. Another example is the enveloping algebra of $\mathfrak{s l}(2, k)$ over a field $k$ of positive characteristic $p$; in this case $H:=U(\mathfrak{s l}(2, k))$ is unimodular, that is $\int_{H}^{l} \cong k$, [BZ, Proposition 6.3(e)]. However it is possible that the equality (e) holds for all regular affine PI Hopf domains of GK-dimension 2.

At this point we can list all the hypotheses that we will use for our classification. By the above theorem, given (a), (d) and (e) in the list below, the map $\pi: H \rightarrow$ $H /$ r.ann $\left(\int_{H}^{l}\right)$ satisfies (b) and (c).

Hypotheses. (a) $H$ is an affine, noetherian, prime, PI Hopf algebra;

(b) there is an algebra homomorphism $\pi: H \rightarrow k$ such that the order $n$ of $\pi$ in $G\left(H^{\circ}\right)$ is equal to the PI-degree of $H$;

(c) the invariant subring $H_{0}^{l}$ under the action of the left winding automorphism $\Xi_{\pi}^{l}$ is a Dedekind domain;

(d) when $p=$ char $k>0$, then $n$ is coprime to $p$;

(e) $\operatorname{GKdim} H=\operatorname{gldim} H=1$.

We remark for future use that the analogues of Theorems 2.3 and 2.5 remain valid (with the same proofs) for any map $\pi$ satisfying the above hypotheses.

\section{EXAmples}

In this section we assemble some examples of prime affine Hopf $k$-algebras of GK-dimension one. Note that we've already listed one such example in Remarks $2.3 \mathrm{~B}(\mathrm{a})$.

3.1. Commutative examples. Recall $\mathrm{Hu}$, Theorem 20.5] that, over an algebraically closed field $k$, there are precisely two connected algebraic groups of dimension one. Therefore there are precisely two commutative $k$-affine domains of GK-dimension one which admit a structure of Hopf algebra, namely $A_{1}=k[X]$ and $A_{2}=k\left[X^{ \pm 1}\right]$. For $A_{1}, \epsilon(X)=0, S(X)=-X$, and

$$
\Delta(X)=X \otimes 1+1 \otimes X
$$

For $A_{2}, \epsilon(X)=1, S(X)=X^{-1}$, and

$$
\Delta(X)=X \otimes X .
$$

Commutativity implies that $i o\left(A_{j}\right)=i m\left(A_{j}\right)=1$ for $j=1,2$. Of course $A_{1}$ and $A_{2}$ are regular, in common with all commutative affine Hopf algebras which are domains, [Wa, Theorem 11.6]. The following result is [LWZ, Theorem 0.2(c)], which takes care of the case when $i o(H)=1$.

Proposition. Suppose $H$ satisfies Hypotheses [2.5. If $\pi: H \rightarrow H / \mathrm{r} \cdot \operatorname{ann}\left(\int_{H}^{l}\right)$ has order 1 (or equivalently, $\int_{H}^{l} \cong k$ as $H$-bimodule), then $H$ is isomorphic as a Hopf algebra to $A_{1}$ or $A_{2}$. 
3.2. Dihedral group algebra. Let $\mathbb{D}$ denote the infinite dihedral group $\langle g, x| g^{2}=$ $\left.1, g x g=x^{-1}\right\rangle$. The group algebra $k \mathbb{D}$ is an affine cocommutative Hopf algebra, which is prime by $[\mathrm{Pa}$, Theorem 4.2.10]. Being a skew group algebra of a finite group over the Laurent polynomial algebra $k\left[x^{ \pm 1}\right], k \mathbb{D}$ has GK-dimension one, [MR, Proposition 8.2.9(iv)]. By [Pa, Theorem 10.3.13], $k \mathbb{D}$ is regular if and only if the characteristic of $k$ is different from 2. By cocommutativity,

$$
i m(k \mathbb{D})=1 .
$$

Since it is a free module of rank 2 over $k\left[x^{ \pm 1}\right], k \mathbb{D} \subseteq M_{2}\left(k\left[x^{ \pm 1}\right]\right)$, so that PI-deg $(k \mathbb{D}) \leq 2$. As the algebra is not commutative,

$$
\mathrm{PI}-\operatorname{deg}(k \mathbb{D})=2 .
$$

Either by direct calculation, or using [BZ, Lemma 6.6], one sees that, as a right module,

$$
\int_{k \mathbb{D}}^{l} \cong k \mathbb{D} /\langle x-1, g+1\rangle .
$$

Case 1: Suppose that the characteristic of $k$ is not 2. It follows from (3.2.1) that $G_{\pi}^{l}=G_{\pi}^{r}=\{1, \tau\} \cong C_{2}$, where $\tau(x)=x$ and $\tau(g)=-g$. Thus the classical components (necessarily equal on account of cocommutativity), are the elements fixed by $\tau$, namely

$$
(k \mathbb{D})_{0}=(k \mathbb{D})_{0}^{l}=(k \mathbb{D})_{0}^{r}=k\left[x^{ \pm 1}\right] .
$$

Finally, $i o(k \mathbb{D})=2$, and $J_{i q}=(x-1) k \mathbb{D}$, so that $(k \mathbb{D})_{i q}=k\langle g\rangle$, the group algebra of the cyclic group of order 2 .

Case 2: Now let $k$ have characteristic 2. Then (3.2.1) shows that $k \mathbb{D}$ is unimodular, so $G_{\pi}^{r}=G_{\pi}^{l}=\{1\}$. Thus $i o(k \mathbb{D})=1$, and the classical components all equal $k \mathbb{D}$, with $J_{i q}$ equal to the augmentation ideal and $(k \mathbb{D})_{i q}=k$.

3.3. Taft algebras. [LWZ, Examples 2.7, 7.3] Let $n$ and $t$ be integers with $n$ greater than 1, and coprime to the characteristic of $k$ if the latter is positive, and with $0 \leq t \leq n-1$. Fix a primitive root $n$th $\operatorname{root} \xi$ of 1 in $k$. Let $H:=H(n, t, \xi)$ be the $k$-algebra generated by $x$ and $g$ subject to the relations

$$
g^{n}=1, \quad \text { and } \quad x g=\xi g x .
$$

Then $H$ is a Hopf algebra with coalgebra structure given by

$$
\Delta(g)=g \otimes g, \epsilon(g)=1, \quad \text { and } \quad \Delta(x)=x \otimes g^{t}+1 \otimes x, \epsilon(x)=0,
$$

and with

$$
S(g)=g^{-1} \text { and } S(x)=-x g^{-t} .
$$

Thus one can think of $H(n, t, \xi)$ either as a (slightly generalised) factor of the quantum Borel of $\mathfrak{s l}(2, k)$ at an $n$th root of unity [BG2, I.3.1], or as a limit of a family of finite dimensional Taft algebras, [Mo, 1.5.6].

Viewing $H(n, t, \xi)$ as the skew group algebra $k[x] *\langle g\rangle$ lets us see easily that it is affine noetherian, prime, hereditary, and has GK-dimension one [LWZ]. An easy calculation shows that $Z(H)=k\left[x^{n}\right]$, so that

$$
\text { PI-deg }(H(n, t, \xi))=n \text {. }
$$

We leave to the reader the routine proof of the 
Proposition. Let $n, n^{\prime}, t, t^{\prime}$ be integers with $n, n^{\prime}$ greater than 1 , and coprime to the characteristic of $k$ if the latter is positive, and with $0 \leq t, t^{\prime} \leq n-1$. Let $\xi$ and $\eta$ be two primitive nth roots of 1 and $\xi^{\prime}$ a primitive $n^{\prime}$ th root of 1 .

(a) As algebras, $H(n, t, \xi) \cong H\left(n^{\prime}, t^{\prime}, \xi^{\prime}\right)$ if and only if $n=n^{\prime}$.

(b) As bialgebras, $H(n, t, \xi) \cong H\left(n^{\prime}, t^{\prime}, \xi\right)$ if and only if $n=n^{\prime}$ and $t=t^{\prime}$.

(c) If $\xi=\eta^{v}$ for some $1 \leq v \leq n-1$, then $H(n, t, \xi) \cong H(n, v t, \eta)$ as Hopf algebras.

Recall that $H=H(n, t, \xi)$. Using [BZ, Lemma 6.6] we find that

$$
\int_{H}^{l} \cong H /\left\langle x, g-\xi^{-1}\right\rangle
$$

The corresponding homomorphism $\pi$ yields left and right winding automorphisms

$$
\Xi_{\pi}^{r}:\left\{\begin{array}{l}
x \mapsto \xi^{-t} x \\
g \mapsto \xi^{-1} g
\end{array} \quad \text { and } \quad \Xi_{\pi}^{l}:\left\{\begin{array}{l}
x \mapsto x \\
g \mapsto \xi^{-1} g,
\end{array}\right.\right.
$$

so that $G_{\pi}^{l}=\left\langle\Xi_{\pi}^{l}\right\rangle$ and $G_{\pi}^{r}=\left\langle\Xi_{\pi}^{r}\right\rangle$ have order $n$. Hence, as predicted by Theorem 2.5(e),

$$
i o(H(n, t, \xi))=n .
$$

The integral annihilator $J_{i q}$ is $\cap\left\{\left\langle x, g-\xi^{i}\right\rangle: 0 \leq i \leq n-1\right\}$, which equals $x H$. Thus $H_{i q}=H / J_{i q} \cong k\langle g\rangle$. As for the right and left classical components,

$$
H_{0}^{r}=H_{\pi}^{G_{\pi}^{r}}=k\left[x g^{-t}\right] \text {, and } H_{0}^{l}=H^{G_{\pi}^{l}}=k[x],
$$

so one checks that, as predicted by Proposition2.1(c), $H_{0}^{r}$ is a left coideal subalgebra of $H$ and $H_{0}^{l}$ is a right coideal subalgebra.

Let $m:=n / \operatorname{gcd}(n, t)$. Then $G_{\pi}^{l} \cap G_{\pi}^{r}=\left\langle\left(\Xi_{\pi}^{l}\right)^{m}\right\rangle$, so that the integral minor of $H(n, t, \xi)$ is

$$
i m(H(n, t, \xi))=m \text {. }
$$

This shows that $G_{\pi} \cong C_{n} \times C_{m}$. Now the classical component

$$
H_{0}=H^{G_{\pi}}=H_{0}^{l} \cap H_{0}^{r}=k\left[x^{m}\right],
$$

and since this is a Hopf subalgebra of $H$ we find that $\widetilde{H_{0}}$, the largest Hopf subalgebra of the classical component, is $H_{0}=k\left[x^{m}\right]$. Note that

$$
Z(H)=k\left[x^{n}\right] \subseteq k\left[x^{m}\right]=\widetilde{H_{0}},
$$

so that $\widetilde{H_{0}}$ is central if and only if the integral minor is $n$ - that is, if and only if $\operatorname{gcd}(n, t)=1$. Nevertheless, $\widetilde{H_{0}}$ can easily be checked to be normal in $H$, and to be a maximal commutative Hopf subalgebra of $H$.

3.4. Generalised Liu algebras. We start with a presentation of generalised Liu algebras that is convenient for the proof of some algebraic properties and then convert it into another form in which the generators of the algebra are more rigid. Both presentations are helpful in understanding these algebras.

Let $n$ be an integer greater than 1 , with $n$ coprime to the characteristic $p$ of $k$ if $p>0$. Fix a primitive $n$th root $\theta$ of one in $k$. Let $w$ be a positive integer with $\operatorname{gcd}(n, w):=b \geq 1$. Write $n=n^{\prime} b, w=w^{\prime} b$. Define a $k$-algebra $B$ with generators $h^{ \pm 1}, f$ and $y$, and relations

$$
h h^{-1}=h^{-1} h=1, \quad f^{b}=1, \quad h f=f h ;
$$




$$
\begin{aligned}
y h & =\theta h y ; \\
y f & =\theta^{n^{\prime}} f y ; \\
y^{n}-1+h^{n w^{\prime}} & =0 .
\end{aligned}
$$

We shall show that $B$ admits a structure of Hopf algebra, but first we develop some of its properties as a $k$-algebra. Start with the group algebra

$$
A:=k\left\langle h^{ \pm 1}, f\right\rangle \cong k\left(C_{\infty} \times C_{b}\right) .
$$

Let $\sigma$ be the $k$-algebra automorphism of $A$ defined by $\sigma(h)=\theta h, \sigma(f)=\theta^{n^{\prime}} f$, and form the skew polynomial algebra

$$
C:=A[y ; \sigma] .
$$

Since $|\sigma|=n$ and $A$ is commutative, $C$ has PI-degree at most $n$. One easily confirms that $A^{\sigma}$ is the group algebra $k\langle x\rangle$, where

$$
x:=h^{n^{\prime}} f^{-1},
$$

so that

$$
Z(C)=k\left[x^{ \pm 1}, y^{n}\right]
$$

Thus $y^{n}-1+x^{w} \in Z(C)$, so that we can form

$$
B:=C /\left\langle y^{n}-1+x^{w}\right\rangle .
$$

Abusing notation, we continue to write $h, f, y, x$ for the images of these elements in $B$. Thus, in $B$,

$$
y^{n}=1-x^{w}=1-h^{n^{\prime} w}=1-h^{n w^{\prime}} .
$$

So $B$ has $k$-basis

$$
\left\{h^{i} f^{j} y^{l}: i \in \mathbb{Z}, 0 \leq j \leq b-1,0 \leq l \leq n-1\right\} .
$$

This shows that

$$
B \text { is a finitely generated free (left and right) } k\left\langle h^{ \pm 1}\right\rangle \text {-module, }
$$

which permits us to confirm firstly that

$$
k\left\langle h^{ \pm 1}\right\rangle \backslash\{0\} \text { consists of regular elements of } B,
$$

and hence, in view of (3.4.2),

$y$ is not a zero divisor in $B$.

Finally, it follows from (3.4.2) and (3.4.3) that

$$
Z(B)=k\left[x^{ \pm 1}\right] .
$$

It's clear from its construction that $B$ is a noetherian algebra with $\operatorname{GKdim}(B)=1$.

We turn now to the coalgebra structure of $B$. For the counit, define

$$
\epsilon(f)=\epsilon(h)=1, \quad \epsilon(y)=0 ;
$$

trivially, this is an algebra homomorphism. For the coproduct, we propose to define

$$
\begin{aligned}
& \Delta(h)=h \otimes h, \quad \Delta(f)=f \otimes f, \\
& \Delta(y)=y \otimes g+1 \otimes y,
\end{aligned}
$$

for a suitable choice of a group-like element $g \in A=\langle h, f\rangle$. Since $g$ is group-like, $g=h^{a} f^{b}$ for some integers $a, b$. Now we require

$$
\Delta(y)^{n}=\Delta\left(y^{n}\right)=\Delta\left(1-x^{w}\right)=\left(1-x^{w}\right) \otimes x^{w}+1 \otimes\left(1-x^{w}\right) .
$$


Thus, by the $q$-binomial theorem [BG2, I.6.1], we require

$$
g^{n}=x^{w}=h^{w^{\prime} n} \quad \text { and } \quad \sigma(g)=\xi g \text { for a primitive } n \text {th } \operatorname{root} \xi \text { of } 1 .
$$

The first of these constraints implies that $g=h^{w^{\prime}} f^{i}$ for some $i, 0 \leq i \leq b-1$. Then $\sigma(g)=\theta^{w^{\prime}+n^{\prime} i} g$ so the second part of (3.4.8) requires that

$$
\operatorname{gcd}\left(n, w^{\prime}+n^{\prime} i\right)=1 \text {. }
$$

By the elementary lemma at the end of this subsection, such a solution $i=i_{0}$ to (3.4.9) exists, and accordingly we set

$$
g:=h^{w^{\prime}} f^{i_{0}}
$$

in the definition of $\Delta(y)$. With these definitions it is routine to check that $\Delta$ is an algebra homomorphism, that it satisfies the coassociativity axioms, and that $\epsilon$ is a counit. Finally, we define

$$
S(h)=h^{-1}, \quad S(f)=f^{-1}, \quad S(y)=-y g^{-1}
$$

and check easily that this extends to an anti-automorphism of $B$ satisfying the antipode axiom.

We show next that the algebra $B$ is also generated by $x^{ \pm 1}, g, y$. Since $\operatorname{gcd}\left(w^{\prime}+\right.$ $\left.n^{\prime} i_{0}, n\right)=1$, there are integers $u$ and $v$ such that $u\left(w^{\prime}+n^{\prime} i_{0}\right)+v n=1$. Then we calculate that

$$
g^{u+v n} x^{\left(u i_{0}-v b\left(w^{\prime}-1\right)\right)}=\left(h^{w^{\prime}} f^{i_{0}}\right)^{u+v n}\left(h^{n^{\prime}} f^{-1}\right)^{\left(u i_{0}-v b\left(w^{\prime}-1\right)\right)}=h .
$$

Hence $h$ is generated by $g$ and $x^{ \pm 1}$, noting that $g^{-1}=g^{n-1} x^{-w}$. Since $f=x^{-1} h^{n^{\prime}}$, $B$ is generated by $y, g$ and $x^{ \pm 1}$. Combining the above assertions, $B$ is generated by $x^{ \pm 1}, g, y$, subject to the relations

$$
\begin{cases}y g=\xi g y, & y^{n}=1-x^{w}=1-g^{n} \\ x y=y x, & x g=g x \\ x x^{-1}=1 & \end{cases}
$$

where $\xi=\theta^{w^{\prime}+n^{\prime} i_{0}}$ is a primitive $n$th root of 1 . The coalgebra structure of $B$ is determined by the fact that $x$ and $g$ are group-like and $y$ is $(g, 1)$-primitive. The Hopf algebra structure of $B$ is uniquely determined by these data and therefore $B$ is denoted by $B(n, w, \xi)$.

Note that two different solutions $i_{0}$ to (3.4.9) give distinct $\xi:=\theta^{w^{\prime}+n^{\prime} i_{0}}$ and different formulas of $g$ in terms of $h$ and $f$ (3.4.10). And, replacing $h$ by $h f^{j}$ for some integer $j$ satisfying $\operatorname{gcd}\left(1+j n^{\prime}, n\right)=1$ changes one $i_{0}$ to another. This means that two different choices of the pair $\left(\theta, i_{0}\right)$ may yield the same Hopf algebra $B$.

One advantage of the second presentation of $B$ (see (3.4.12) ) is that the ordered set $\{n, w, \xi, x, g\}$ can be recovered uniquely by the Hopf algebra structure of $B$ (see (j) of the next theorem). In this first presentation, however, $h$ (and $\theta$ ) can not be determined uniquely by the Hopf structure of $B$.

We are now ready to list the main properties of the algebras $B=B(n, w, \xi)$ :

Theorem. Let the $k$-algebra $B=B(n, w, \xi)$ be defined as above. Assume that the characteristic of $k$ does not divide $n$.

(a) As a $k$-algebra, $B$ is generated by $y, h^{ \pm 1}, f$, with relations as stated at the beginning of this section; and it is also generated by $y, g$ and $x^{ \pm 1}$, with relations in (3.4.12). 
(b) B is an affine noetherian Hopf algebra of Gel'fand-Kirillov dimension 1, with center $k\left\langle x^{ \pm 1}\right\rangle$.

(c) $B$ is prime.

(d) $\mathrm{PI}-\operatorname{deg}(B)=n$.

(e) $B$ has finite global dimension if and only if $w$ is a unit in $k$. In this case, gldim $B=1$.

(f) $i o(B)=i m(B)=n$.

(g) The fixed subring $B^{\Xi_{\pi}^{l}}$ is $k\left\langle y, x^{ \pm 1}\right\rangle$; and $B^{\Xi_{\pi}^{r}}=k\left\langle y g^{-1}, x^{ \pm 1}\right\rangle$. Thus

$$
B_{0}:=B^{\Xi_{\pi}^{l}} \cap B^{\Xi_{\pi}^{r}}=k\left[x^{ \pm 1}\right]=Z(B) .
$$

(h) The group of group-like elements of $B$ is $\langle h, f\rangle$, isomorphic to $C_{\infty} \times C_{b}$.

(i) If $z \in B$ is a nonzero $(a, 1)$-primitive element for some group-like element $a \neq 1$, then $a=g$ and $z \in k y+k(1-g)$.

(j) Let $B(\bar{n}, \bar{w}, \bar{\xi})$ be another Hopf algebra of the same kind with generators $\bar{x}^{ \pm 1}, \bar{g}$, and $\bar{y}$. If $\phi: B(\bar{n}, \bar{w}, \bar{\xi}) \rightarrow B(n, w, \xi)$ is a Hopf algebra isomorphism, then $n=\bar{n}, w=\bar{w}, \xi=\bar{\xi}$ and $\phi(\bar{x})=x, \phi(\bar{g})=g, \phi(\bar{y})=\eta y$ for some $n$th root $\eta$ of 1 .

Proof. (a) and (b) are clear from the discussion above.

(c) In view of (3.4.5), to show that $B$ is prime it suffices to prove that, if $I$ is a non-zero ideal of $B$, then

$$
I \cap k\left\langle h^{ \pm 1}\right\rangle \neq 0 .
$$

So let $\alpha$ be a non-zero element of $I$. Using (3.4.3) we can uniquely write

$$
\alpha=\sum_{l=0}^{n-1} \alpha_{l} y^{l}
$$

with $\alpha_{l} \in k\left\langle h^{ \pm 1}, f\right\rangle$ and choose $\alpha$ so that the number of non-zero $\alpha_{l}$ occurring is as small as possible for non-zero elements of $I$. We assume for a contradiction that this number is greater than one. Since $y^{n}=1-h^{n w^{\prime}}$, we can multiply $\alpha$ on the right by a suitable power of $y$ to ensure that $\alpha_{0} \neq 0$. Now $\alpha h-h \alpha \in I$ is non-zero and has strictly smaller $y$-length than $\alpha$, contradicting our choice of $\alpha$. Therefore

$$
I \cap k\left\langle h^{ \pm 1}, f\right\rangle \neq 0 .
$$

Now $\theta^{n^{\prime}}$ is a primitive $b$ th root of 1 in $k$, so the $b$ primitive idempotents

$$
e_{j}:=\frac{1}{b} \sum_{i=0}^{b-1} \theta^{n^{\prime} j i} f^{i}
$$

form a $k$-basis of $k\langle f\rangle$. Fix

$$
0 \neq \beta=\sum_{j=0}^{b-1} \gamma_{j} e_{j} \in I \cap k\left\langle h^{ \pm 1}, f\right\rangle .
$$

There exists $j$ such that $0 \neq \beta e_{j}=\gamma_{j} e_{j}$. That is, relabelling, there exists $j$ with $0 \leq j \leq b-1$ and $0 \neq \gamma \in k\left\langle h^{ \pm 1}\right\rangle$ such that $\gamma e_{j} \in I$. Notice now that $e_{j} y=y e_{j-1}$. It follows that, for all $t=0, \ldots, b-1$,

$$
I \ni y^{n-t} \gamma e_{j} y^{t}=y^{n} \gamma_{t} e_{j-t}=\left(1-h^{n w^{\prime}}\right) \gamma_{t} e_{j-t},
$$

where $0 \neq \gamma_{t} \in k\left\langle h^{ \pm 1}\right\rangle$. Multiplying these $b$ non-zero elements of $I$ on the left by suitable elements of the commutative domain $k\left\langle h^{ \pm 1}\right\rangle$, we find that there exists 
$0 \neq c \in k\left\langle h^{ \pm 1}\right\rangle$ such that $c e_{j-t} \in I$ for all $t=0, \ldots, b-1$. Adding these elements, (3.4.13) follows.

(d) Since $B$ is prime by (c) we can work in the simple artinian quotient ring $Q(B)$ of $B$. Now $Q(Z(B))=Z(Q(B))=k(x)$, and we see from the definition (3.4.1) of $x$ and the $k$-basis (3.4.3) that

$$
\operatorname{dim}_{Q(Z)}(Q(B))=n^{\prime} b n=n^{2} .
$$

This proves (d).

(e) Suppose first that $w$ is a unit in $k$. by [LL, Corollary 2.4] it suffices to prove that the trivial $B$-module $k$ has projective dimension 1. Noting (3.4.6) that $y$ is a normal regular element and setting $D:=B / y B, D$ has $k$-basis $\left\{h^{i} f^{j}: 0 \leq i \leq\right.$ $\left.n w^{\prime}-1,0 \leq j \leq b-1\right\}$, so that

$$
D \cong k\left(C_{n^{\prime} w} \times C_{b}\right) \text {. }
$$

it follows that the group algebra $D$ of (3.4.14) is semisimple Artinian, so we have that $\operatorname{prdim}_{B}(k)=1$ as required.

Now suppose that $p \mid w$, so that $p \mid w^{\prime}$. Suppose for a contradiction that $B$ has finite global dimension. It is clear from (3.4.4) that $B$ is a free module over the commutative algebra $R:=k\left\langle h^{ \pm n}, y\right\rangle$, and therefore, by restricting $B$-resolutions to $R$-resolutions we deduce that $R$ has finite global dimension. But from (3.4.2) we see that, as a commutative $k$-algebra,

$$
R \cong k\left\langle U^{ \pm 1}, V: U^{w^{\prime}}=1-V^{n}\right\rangle,
$$

and the Jacobian Criterion shows that $R$ is singular. Thus $B$ also has infinite global dimension.

(f) By [LWZ, Lemma 2.6], the left homological integral $\int_{B}^{l}$ of $B$ is the the 1dimensional right module $B /\left\langle y, h-\theta^{-1}, f-\theta^{-n^{\prime}}\right\rangle$. Hence the left and right winding automorphisms associated to $\int_{B}^{l}$ are

$$
\Xi_{\pi}^{l}:\left\{\begin{array}{l}
y \mapsto y, \\
h \mapsto \theta^{-1} h, \\
f \mapsto \theta^{-n^{\prime} f}
\end{array} \quad \text { and } \quad \Xi_{\pi}^{r}:\left\{\begin{array}{l}
y \mapsto \theta^{-\left(w^{\prime}+n^{\prime} i_{0}\right)} y, \\
h \mapsto \theta^{-1} h, \\
f \mapsto \theta^{-n^{\prime}} f .
\end{array}\right.\right.
$$

Clearly these automorphisms have order $n$, whence $i o(B)=n$. Moreover our choice of $i_{0}$ as a solution of (3.4.9) shows that $G_{\pi}^{l} \cap G_{\pi}^{r}=\{1\}$. This proves (f).

(g) This follows easily from the descriptions in (f) of the automorphisms.

(h) It's clear that $k\langle h, f\rangle$ is the biggest sub-Hopf algebra of $B$ which is a group algebra, so this follows from the linear independence of distinct group-like elements [Sw, 3.2.1].

(i) By (3.4.3), $z=\sum_{i=0}^{n-1} z_{i} y^{i}$ where $z_{i} \in A:=k\left\langle x^{ \pm 1}, g\right\rangle=k\left\langle h^{ \pm 1}, f\right\rangle$. Pick $r$ such that $z_{r} \neq 0$ and $z_{i}=0$ for all $r<i \leq n-1$. Hence

$$
\begin{aligned}
\Delta(z) & =\sum_{i=0}^{r} \Delta\left(z_{i}\right) \Delta\left(y^{i}\right)=\sum_{i=0}^{r} \Delta\left(z_{i}\right) \Delta(y)^{i} \\
& =\sum_{i=0}^{r} \Delta\left(z_{i}\right)(y \otimes g+1 \otimes y)^{i} \\
& =\sum_{i=0}^{r} \Delta\left(z_{i}\right)\left(\sum_{s=0}^{i} y^{s} \otimes\left(\begin{array}{l}
i \\
s
\end{array}\right)_{\xi} g^{s} y^{i-s}\right) .
\end{aligned}
$$


Since $z$ is a $(a, 1)$-primitive,

$$
\Delta(z)=z \otimes a+1 \otimes z=\left(\sum_{i=0}^{r} z_{i} y^{i}\right) \otimes a+1 \otimes\left(\sum_{i=0}^{r} z_{i} y^{i}\right) .
$$

If $r \geq 2$, by comparing the terms of $y$-degree $(1, r-1)$ in two expressions of $\Delta(z)$ and using (3.4.3), we have

$$
\Delta\left(z_{r}\right)\left(y \otimes\left(\begin{array}{l}
r \\
1
\end{array}\right) g_{\xi} g^{1} y^{r-1}\right)=0,
$$

which implies that $\Delta\left(z_{r}\right)=0$, and hence $z_{r}=0$ after applying $\epsilon \otimes 1$. This contradicts the choice of $r$. Therefore $z=z_{0}+z_{1} y$. By $(a, 1)$-primitiveness of $z$,

$$
\Delta\left(z_{0}\right)+\Delta\left(z_{1}\right)(y \otimes g+1 \otimes y)=\left(z_{0}+z_{1} y\right) \otimes a+1 \otimes\left(z_{0}+z_{1} y\right),
$$

which implies that

$$
\Delta\left(z_{0}\right)=z_{0} \otimes a+1 \otimes z_{0}, \quad \Delta\left(z_{1}\right)(1 \otimes g)=z_{1} \otimes a, \quad \Delta\left(z_{1}\right)=1 \otimes z_{1} .
$$

By using the coalgebra axioms several times, we obtain

$$
z_{1}=c_{1}, \quad a=g, \quad z_{0}=c_{2}(1-g)
$$

for some $c_{1}, c_{2} \in k$, as desired.

(j) By (d) $n=\bar{n}$. By (b), $\phi(\bar{x})$ is either $x$ or $x^{-1}$. Let $z=\phi(\bar{y})$ and $a=\phi(\bar{g})$. Since $\bar{y}$ is $(\bar{g}, 1)$-primitive, $z$ is $(a, 1)$-primitive. By (i), $a=g$ and $z=c_{1} y+c_{2}(1-g)$. Since $\{\bar{y}, \bar{g}\}$ are skew-commutative, so is the pair $\{z, a=g\}$. This implies that $c_{2}=0$. The relation $y^{n}=1-g^{n}$ implies that $c_{1}=\eta$ is an $n$th root of 1 and hence $\phi(\bar{y})=\eta y$. By the relations in (3.4.12), we have

$$
g^{n}=\phi\left(\bar{g}^{n}\right)=\phi\left(1-\bar{x}^{\bar{w}}\right)=1-x^{ \pm \bar{w}}
$$

and

$$
g^{n}=1-x^{w} .
$$

This forces $w=\bar{w}$ and $\phi(\bar{x})=x$. Finally, $\xi=\bar{\xi}$ follows from the first relation in (3.4.12) and the facts that $\eta y=\phi(\bar{y})$ and $g=\phi(\bar{g})$.

Remarks. Suppose that $\operatorname{gcd}(n, w)=b=1$ in the above construction. Then the group of group-like elements of $B$ is $\langle h\rangle$, infinite cyclic, so $B=k\langle h, y\rangle$ is a Hopf algebra of the class constructed by Liu [Liu. Indeed it's clear that all of Liu's examples arise in this way, precisely as those algebras $B(n, w, \xi)$ with $\operatorname{gcd}(n, w)=1$.

Here is the lemma needed to find a solution $i$ to (3.4.9).

Lemma. Let $n$ and $w$ be positive integers with $\operatorname{gcd}(n, w)=b$, and write $n=$ $b n^{\prime}, w=b w^{\prime}$. There exists $i, 0 \leq i \leq b-1$, such that $\operatorname{gcd}\left(n, w^{\prime}+n^{\prime} i\right)=1$.

Proof. Define $i_{0}:=\prod\left\{p: p\right.$ prime, $\left.p \mid b, p \nmid w^{\prime}\right\}$, with $i_{0}=1$ if there are no primes in the indicated set. We claim that $\operatorname{gcd}\left(n, w^{\prime}+n^{\prime} i_{0}\right)=1$. Let $q$ be a prime divisor of $n$. If (i) $q \mid n^{\prime}$ then $q \nmid w^{\prime}$, so $q \nmid\left(w^{\prime}+n^{\prime} i_{0}\right)$. Suppose (ii) that $q \nmid n^{\prime}$, so that $q \mid b$. If $q \mid w^{\prime}$, then $q \nmid i_{0}$ and so $q \nmid n^{\prime} i_{0}$. Therefore $q \nmid\left(w^{\prime}+n^{\prime} i_{0}\right)$. If $q \nmid n^{\prime}$, so that $q \mid b$, but $q \nmid w^{\prime}$, then $q \mid i_{0}$, and hence $q \nmid\left(w^{\prime}+n^{\prime} i_{0}\right)$. Combining (i) and (ii) yields the result. 


\section{Classification I: $\operatorname{im}(H)=1$}

4.1. Primitive versus group-like. Having dealt in Proposition 3.1 with the case where $i o(H)=1$, henceforth we shall always assume that

$$
n:=i o(H)>1 \text {. }
$$

We begin the classification proof by dealing first with the subclass of prime affine regular Hopf algebras $H$ of GK-dimension one for which

$$
i m(H)=1 \text {. }
$$

In fact, in a small (and, as we shall see eventually, purely formal) weakening of this assumption, it will be convenient to assume that

there exists an algebra map $\pi: H \rightarrow k$ satisfying both Hypotheses

2.5 and the condition

$$
\left|G_{\pi}^{l} /\left(G_{\pi}^{l} \cap G_{\pi}^{r}\right)\right|=1
$$

Notice that (4.1.2) implies that $G_{\pi}^{l}=G_{\pi}^{r}$ and hence $H_{\pi}^{l}=H_{\pi}^{r}=H_{\pi}=\widetilde{H_{\pi}}$, the latter being a subHopf algebra of $H$, by Proposition [2.1(h). In particular, cocommutative Hopf algebras satisfy (4.1.1) and (4.1.2); conversely, it follows from the theorem we prove below that every Hopf algebra $H$ satisfying Hypotheses 2.5 and (4.1.2) is cocommutative.

More precisely, we shall prove

Theorem. Suppose $(H, \pi)$ satisfies Hypotheses 2.5 and (4.1.2). Then $H$ is isomorphic as a Hopf algebra either to the Taft algebra $H(n, 0, \xi)$ of Example 3.3, or to the dihedral group algebra $k \mathbb{D}$ of Example 3.2. As a consequence, $H$ is cocommutative and satisfies (4.1.1).

The conclusion that cocommutativity follows from (4.1.2) is not valid when the GK-dimension is bigger than one - for example $\mathcal{U}:=\mathcal{U}_{\epsilon}\left(\mathfrak{s l}_{2}\right)$ is an affine regular domain and a Hopf algebra, which is PI with GK-dimension 3. In this case, $i o(\mathcal{U})=$ $i m(\mathcal{U})=1$, but $\mathcal{U}$ is not cocommutative. But we don't seem to know what happens in GK-dimension 2.

The dichotomy appearing in the following definition will feature heavily throughout the rest of the paper.

Definition. Let $H$ be a prime affine regular Hopf algebra of GK-dimension one. Assume Hypotheses 2.5 and (4.1.2). By Proposition 2.1(h), together with Theorem 2.5(f) and Examples [3.1, $H_{\pi}$ is isomorphic as a Hopf algebra either to $k[x]$ or to $k\left[x^{ \pm 1}\right]$.

(a) $H$ is called primitive if $H_{\pi}=k[x]$.

(b) $H$ is called group-like if $H_{\pi}=k\left[x^{ \pm 1}\right]$.

We shall see from our final result that this definition is independent of the choices of $\pi$ under Hypotheses 2.5 .

\subsection{Primitive case.}

Proposition. Suppose $(H, \pi)$ satisfies Hypotheses 2.5 and (4.1.2). If $H$ is primitive, then $H$ is isomorphic as a Hopf algebra to the Taft algebra $H(n, 0, \xi)$ of Example 3.3 . 
Proof. Since $\widehat{G_{\pi}^{l}}=\mathbb{Z}_{n}=\{0,1, \cdots, n-1\}$, we write $H_{\pi, i}^{l}=H_{i}^{l}$ for all $i \in \widehat{G_{\pi}^{l}}$. Similarly define $H_{i}^{r}$. By (4.1.2), we have

$$
H_{0}^{l}=H_{\pi}^{l}=H_{\pi}^{r}=H_{0}^{r}
$$

which is also equal to $H_{0}$ (and $\widetilde{H_{0}}$ ).

Since $H$ is primitive, $H_{0}=k[x]$ and it is a Hopf subalgebra by Proposition 2.1(h), after a linear change of variable we may assume

$$
\Delta(x)=x \otimes 1+1 \otimes x, \quad S(x)=-x \text { and } \epsilon(x)=0 .
$$

By Proposition 2.2, $H=\oplus_{i \in \mathbb{Z}_{n}} H_{i}^{l}$, with each $H_{i}^{l}$ an invertible module over $H_{0}^{l}=$ $k[x]$. Hence, for each $i, H_{i}^{l}=u_{i} k[x]=k[x] v_{i}$ for some $u_{i}, v_{i} \in H_{i}^{l}$. Fix $\alpha, \delta \in k[x]$ so that $u_{i}=\alpha v_{i}$ and $v_{i}=u_{i} \delta$. Then $k[x] u_{i} \subseteq u_{i} k[x]$, so that $u_{i}=\alpha u_{i} \delta=u_{i} \beta \delta$ for some $\beta \in k[x]$. Thus $\delta$ is a unit of $k[x]$ and so $H_{i}^{l}=u_{i} k[x]=k[x] u_{i}$. Now the strong grading condition implies that $u_{i} u_{-i}$ is a unit element in $H_{0}^{l}=k[x]$. Hence each $u_{i}$ is a unit element. Set $g=u_{1}$. Then we may take $u_{i}:=g^{i}$ for all $i, 0 \leq i<n$, so that $H$ is generated as a $k$-algebra by $x$ and $g$. Now $g^{n}$ equals a non-zero scalar in $H_{0}^{l}=k[x]$, so, since $k$ contains primitive $n$th roots thanks to Hypotheses 2.5(d), we can adjust our choice of $g$ if necessary so that $g^{n}=1$. This in turn forces $\epsilon(g)$ to be an $n$th root of 1 in $k$, so, possibly after another adjustment, we can assume that

$$
\epsilon(g)=1 \text {. }
$$

Fix $i, 1 \leq i \leq n-1$. Since, by (4.1.2), $G_{\pi}^{l}=G_{\pi}^{r}, H_{i}^{l}=H_{j}^{r}$ for some $j$, $1 \leq j \leq n-1$. That is, there is a permutation $i \mapsto i^{\prime}$ of $\{1, \ldots, n-1\}$ such that $H_{i}^{\bar{l}}=\bar{H}_{i^{\prime}}^{r}$.

In particular, by Proposition and Remark 2.2 (a),

$$
\Delta(g) \in\left(H_{1}^{l} \otimes H\right) \cap\left(H \otimes H_{1^{\prime}}^{r}\right)=H_{1}^{l} \otimes H_{1^{\prime}}^{r}=H_{1}^{l} \otimes H_{1}^{l} .
$$

So we can write

$$
\Delta(g)=(g \otimes g) q
$$

for some $q \in H_{0} \otimes H_{0}=k[x] \otimes k[x]$. Since $g$ is invertible, so is $q$, and so $q \in k \backslash\{0\}$. Now (4.2.1) and the fact that $m_{A} \circ(i d \otimes \epsilon) \circ \Delta=i d$ force $q=1$. Therefore $g$ is group-like, and so $S(g)=g^{-1}$.

Now $H_{1}^{l}=k[x] g=g k[x]$, so conjugation by $g$ yields an algebra automorphism of $k[x]$. Thus there are $a, b \in k$ with $b \neq 0$ such that

$$
x g=g(a+b x) .
$$

Applying $\epsilon$ to this shows that $a=0$; and, since $g^{n}=1$, we have $b^{n}=1$. Therefore $H$ is a factor of the algebra $C:=k\left\langle x, g: x g=b g x, g^{n}=1\right\rangle$, and both algebras are free of rank $n$ over $k[x]$. Thus $H=C$, and primeness of $H$ entails that $b$ is a primitive $n$th root of 1 . This completes the proof.

\subsection{Group-like case.}

Proposition. Suppose $(H, \pi)$ satisfies Hypotheses 2.5 and (4.1.2). If $H$ is grouplike, then $H$ is isomorphic as a Hopf algebra to the dihedral group algebra $k \mathbb{D}$ of Example 3.2. 
Proof. Since $H$ is group-like, $H_{0}=k\left[x^{ \pm 1}\right]$. The first three paragraphs of the proof of Proposition 4.2 apply mutatis mutandis to the group-like case. We find that, after adjustments, there is an invertible element $g \in H_{1}^{l}$ with $\varepsilon(g)=1$ such that $H_{i}^{l}=g^{i} k\left[x^{ \pm 1}\right]$ for $i=0, \ldots, n-1$. Moreover, there exists $j \in \mathbb{Z}$ with

$$
g^{n}=x^{j} .
$$

And there is a permutation $i \mapsto i^{\prime}$ of $\{1, \ldots, n-1\}$ such that $H_{i}^{l}=H_{i^{\prime}}^{r}$. As in the previous subsection,

$$
\Delta(g)=(g \otimes g) q
$$

for an invertible element $q$ of $H_{0} \otimes H_{0}$. Applying the Hopf axioms as before, we deduce that $q=1$, so that $g$ is a group-like element. Therefore $H$ is generated as a $k$-algebra by the two group-like elements $g$ and $x$, so $H$ is a group algebra. Now it is easy to deduce that, if $n \neq 0$ then $H \cong k \mathbb{D}$ is the only possibility, as in [LWZ, Proposition 8.2(b)].

\section{THE CENTER}

This section is preparatory for $\S 6$, where we deal with the case $i m(H)=i o(H)$ of the classification.

5.1. Abelian strong gradings. We begin with some further analysis of an algebra strongly graded by a finite abelian group, with commutative identity component, a set-up we have been led to in 2.5. Recall that, if $Z$ is an Ore domain, then the rank of a $Z$-module $M$ is defined to be the $Q(Z)$-dimension of $Q(Z) \otimes_{Z} M$; the rank of a right $Z$-module is defined analogously. The PI-degree of an algebra $R$ has been recalled in (2.3).

Proposition. Let $D$ be a k-algebra and $G$ a finite abelian group acting faithfully on D. Suppose that the order $n$ of $G$ is a unit in $k$, so that $D$ is $\widehat{G}$-graded,

$$
D=\oplus_{\chi \in \widehat{G}} D_{\chi} .
$$

Assume that

(i) (5.1.1) is a strong grading, and

(ii) $D_{0}$ is a commutative domain, with field of fractions $Q_{0}$.

Then the following hold.

(a) $D$ is a semiprime Goldie PI-algebra, with PI- $\operatorname{deg}(D) \leq n$. Every nonzero homogeneous element is a regular element of $D$.

(b) There is an action of $\widehat{G}$ on $Q_{0}$. Denote the automorphism of $Q_{0}$ corresponding to $\chi \in \widehat{G}$ by $\kappa_{\chi}$. Then, for every non-zero element $x$ of $D_{\chi}$,

$$
x a=\kappa_{\chi}(a) x
$$

for all $a \in Q_{0}$. For all $\chi \in \widehat{G}, \kappa_{\chi}\left(D_{0}\right) \subseteq D_{0}$; that is, $\kappa_{\chi}$ restricts to an automorphism of $D_{0}$.

(c) Let $K:=\left\{\kappa_{\chi} \mid \chi \in \widehat{G}\right\} \subseteq \operatorname{Aut}\left(D_{0}\right)$. If $L$ is a cyclic subgroup of the kernel of the homomorphism $\kappa: \widehat{G} \rightarrow K: \chi \mapsto \kappa_{\chi}$, then

$$
\text { PI- } \operatorname{deg}(D) \leq|\widehat{G} / L| \text {. }
$$

(d) Consider the following statements:

(1) PI-deg $(D)=n$.

(2) $\widehat{G}$ acts faithfully on $Q_{0}$. 
(3) $\widehat{G}$ acts faithfully on $Q_{0}$ and the center $Z(D) \subseteq D_{0}$.

(4) $D$ is prime.

Then (1), (2) and (3) are equivalent, and they imply (4). (4) does not imply the others. When (1,2,3) hold,

$$
Z(D)=\left\{a \in D_{0} \mid \kappa_{\chi}(a)=a \forall \chi \in \widehat{G}\right\} .
$$

Proof. Thanks to hypotheses (i) and (ii) $D$ is a torsion free module of finite rank over a commutative ring, and so $D$ is a PI-algebra. Since the nilpotent radical $N$ of $D$ is $G$-invariant, it is $\widehat{G}$-graded. Therefore, by strong grading (i), $N=\left(N \cap D_{0}\right) D$, so (ii) forces $N=0$. We claim that

$$
D \text { is a right Goldie ring. }
$$

For, being strongly graded, $D$ is a projective (hence torsion-free) right $D_{0}-$ module. Thus, as right $D_{0}$-module, $D$ embeds in the finite dimensional $Q\left(D_{0}\right)$-vector space $D Q\left(D_{0}\right)$, and (5.1.4) follows easily. Therefore Goldie's theorem MR, Theorem 2.3.6] ensures that $D$ has a semisimple artinian quotient ring $Q$. The $G$-action on $D$ extends to a $G$-action on $Q$, so that $Q$ is a $\widehat{G}$-graded algebra containing $D$ as a graded subalgebra. Since $D$ is strongly $\widehat{G}$-graded, so is $Q, Q=\oplus_{\chi \in \widehat{G}} Q_{\chi}$. In particular, each non-zero element of $Q_{\chi}$ is a unit, and no nonzero element of $D_{\chi}$ is a zero divisor.

For each $\chi \in \widehat{G}, D_{\chi}$ is an invertible $D_{0}$-module, so $Q_{0} D_{\chi}=Q_{0} u_{\chi}$ for any nonzero element $u_{\chi} \in D_{\chi}$. Thus $Q_{0} D=\oplus_{\chi \in \widehat{G}} Q_{0} u_{\chi}$ is a finite dimensional left $Q_{0}$-vector space. Since right multiplication of this space by any non-zerodivisor of $D$ induces an invertible map, $Q_{0} D=Q$. Similarly, $D Q_{0}=Q$. Therefore

$$
Q=\oplus_{\chi \in \widehat{G}} Q_{0} u_{\chi}=\oplus_{\chi \in \widehat{G}} u_{\chi} Q_{0}
$$

as graded spaces. This also shows that $D_{0}-\{0\}$ is an Ore set of $D$ and the associated localization of $D$ is $Q$. Thus we may assume whenever convenient in the rest of the proof that $D_{0}=Q_{0}$ is a field and $D=Q$ is semisimple artinian.

(a) Since $D$ is a subring of $\operatorname{End}\left(Q_{Q_{0}}\right)=\operatorname{End}\left(Q_{0}^{\oplus n}\right) \cong M_{n}\left(Q_{0}\right)$ where $n=|\widehat{G}|$, PI-deg $(D) \leq n$.

(b) For $a \in Q_{0}$, define $\kappa_{\chi}(a)=u_{\chi} a u_{\chi}^{-1}$. Then $\kappa_{\chi}$ is an automorphism of $Q_{0}$ independent of the choice of a nonzero element $u_{\chi} \in Q_{\chi}=Q_{0} u_{\chi}$. The map $\kappa: \chi \rightarrow \kappa_{\chi}$ induces a surjective group homomorphism from $\widehat{G}$ to $K=\left\{\kappa_{\chi} \mid \chi \in \widehat{G}\right\}$.

Let $a \in D_{0}$ and $\chi \in \widehat{G}$. From (5.1.2) with $x$ in $D_{\chi}$ we see that

$$
\kappa_{\chi}(a) D_{\chi} \subseteq D_{\chi} .
$$

Multiplying this inclusion on the right by $D_{-\chi}$ and appealing to strong grading, we deduce that $\kappa_{\chi}(a) D_{0} \subseteq D_{0}$. Thus $\kappa_{\chi}(a) \in D_{0}$ as claimed.

(c) Let $L$ be any cyclic subgroup of the kernel of the map from $\widehat{G}$ to $K$ and let $\chi_{L}$ be the generator of $L$. Let $t$ be the order of $P:=\widehat{G} / L$. For each $\operatorname{coset} w$ in $P$, set

$$
C_{w}=\oplus_{\chi \in w} Q_{\chi}
$$

Then $D=\oplus_{w \in P} C_{w}$, and $C_{0}$ is the commutative subalgebra of $D$ generated by $Q_{0}$ and $u_{\chi_{L}}$. (Note that $u_{\chi_{L}}$ commutes with $Q_{0}$ because $\chi_{L}$ is in the kernel of $\widehat{G} \rightarrow K$.) For each $w \in P$ choose $\chi \in w$ and $0 \neq u_{\chi} \in Q_{\chi}$. Define $u_{w}:=u_{\chi}$, so that

$$
D=\oplus_{w \in P} C_{w}=\oplus_{w \in P} u_{w} C_{0}=\oplus_{i \in P} C_{0} u_{w} .
$$


Therefore $D$ is strongly $P$-graded and it is a subring of $\operatorname{End}\left(D_{C_{0}}\right) \cong M_{t}\left(C_{0}\right)$. Hence, PI-deg $(D) \leq t$.

(d) Suppose (1), that PI-deg $(D)=n$. Then (2) follows from (c). Suppose (2), so that the map from $\widehat{G}$ to $K$ is an isomorphism. The center of $D$ is $\widehat{G}$-graded, and if $0 \neq x \in D_{\chi} \cap Z(D)$ for some $0 \neq \chi \in \widehat{G}$ then (5.1.2) is contradicted. Therefore $Z(D) \subseteq Q_{0}$, and (5.1.3) is clear. Thus (2) implies (3).

Suppose now that (3) holds. Since $K$ acts on $Q_{0}$ faithfully,

$$
Q_{0}=\oplus_{\eta \in \widehat{K}}\left(Q_{0}\right)_{\eta},
$$

where, by Lemma 2.2, each $\left(Q_{0}\right)_{\eta} \neq 0$. By hypothesis $(3)$ and (5.1.3),$Z(Q)=$ $\left(Q_{0}\right)_{0}$. Thus $\operatorname{rank}_{Z(Q)} Q_{0} \geq|K|=n$. Since $Q$ is strongly $\widehat{G}$-graded, $\operatorname{rank}_{Q_{0}} Q=n$. Combining these we have $n^{2} \leq \operatorname{rank}_{Z(Q)} Q$; thus, by (a) and the definition of the PI-degree, PI-deg $(D)=$ PI- $\operatorname{deg}(Q)=n$, showing that (3) implies (1).

Assume now that (1), (2) and (3) hold. Then $Z(D) \subset D_{0}$ is a domain. This implies that $D$ is prime.

Finally, take $D=\mathbb{C}\left[x^{ \pm 1}\right]$ with the group $G$ of order 2 acting by $x \mapsto-x$, to see that (4) does not imply (1).

Corollary. Assume the hypotheses of Proposition [5.1 above and suppose that

$$
\text { PI-deg }(D)=n=|G| \text {. }
$$

Let $K$ be a subgroup of $\widehat{G}$ and $B$ the subalgebra $\oplus_{\chi} \in K=D_{\chi}$

(a) The restriction of the automorphisms in $G$ to $B$ induces a group homomorphism $f: G \rightarrow \operatorname{Aut}_{k \text {-alg }}(B)$ with image $L$ such that $\widehat{L}=K$.

(b) $B$ is prime with $P I$-degree $|K|$.

Proof. (a) It is clear that $G$ acts in $B$. Therefore $L=G / k e r f$ acts on $B$ faithfully. By the hypotheses of Proposition 5.1 $|L|$ is a unit in $k$. Hence $B=\oplus_{\chi \in \widehat{L}} B_{\chi}$. Since every character of $L$ is a character of $G, B_{\chi}=D_{\chi}$ and hence

$$
B=\oplus_{\chi \in \widehat{L}} B_{\chi}=\oplus_{\chi \in \widehat{L}} D_{\chi} \text {. }
$$

By Lemma 2.2, each $D_{\chi} \neq 0$ for all $\chi \in \widehat{L}$. Thus $K=\widehat{L}$.

(b) Since $\widehat{L}=K$ is a subgroup of $\widehat{G}$, it acts on $D_{0}$ faithfully. By Proposition 5.1(d) above, $B$ is prime and PI-deg $(B)=|K|=|L|$.

5.2. The center. We return for the remainder of $\S 5$ to the study of a prime affine regular Hopf $k$-algebra $A$ of GK-dimension one, with $i o(A)=n$. We assume Hypotheses 2.5(a,b,c,d) (but not (e)). We fix any algebra homomorphism $\pi: A \rightarrow k$ of order $n=i o(A)$ as in Hypotheses 2.5(a,b,c,d) and subgroups $G_{\pi}^{l}, G_{\pi}^{r}, G_{\pi}$ of $\operatorname{Aut}_{k-\text { alg }}(A)$, with corresponding fixed rings $A_{0}^{l}, A_{0}^{r}$ and $A_{0}$, as in $\S 2$. There are decompositions

$$
A=\oplus_{i \in \widehat{G_{\pi}^{l}}} A_{i}^{l}=\oplus_{i \in \widehat{G_{\pi}^{r}}} A_{i}^{r},
$$

which are strong gradings thanks to Proposition 2.2 Let $Q_{0}^{l}$ and $Q_{0}^{r}$ denote the fields $Q\left(A_{0}^{l}\right)$ and $Q\left(A_{0}^{r}\right)$ respectively, and let $Q_{0}=Q\left(A_{0}\right)$. As before, $Q$ will denote the simple artinian ring $Q(A)$. We use $\kappa_{\chi}^{l}$ and $\kappa_{\chi}^{r}$ to denote the automorphisms associated to an element $\chi$ from $G_{\pi}^{l}$ or $G_{\pi}^{r}$ respectively, as defined in (5.1.2). These form subgroups $K_{\pi}^{l}:=\left\{\kappa_{\chi}^{l}: \chi \in G_{\pi}^{l}\right\}$ and $K_{\pi}^{r}:=\left\{\kappa_{\chi}^{r}: \chi \in G_{\pi}^{r}\right\}$ of $\operatorname{Aut}\left(A_{0}^{l}\right)$ and $\operatorname{Aut}\left(A_{0}^{r}\right)$ respectively. We aim to compare the actions of two groups on $A_{0}^{l}$ first, since $G_{\pi}^{r}$ and $G_{\pi}^{l}$ commute by Proposition 2.1(d), $G_{\pi}^{r}$ acts on $A_{0}^{l}$. Denote by 
$\rho^{l}$ the resulting map from $G_{\pi}^{r}$ to $\operatorname{Aut}\left(A_{0}^{l}\right)$, bearing in mind that $\rho^{l}$ may not be a monomorphism. The second group acting on $A_{0}^{l}$ is $\widehat{G_{\pi}^{l}}$, acting through the map $\kappa^{l}$ to $K_{\pi}^{l}$ as defined in Proposition 5.1(b). By Hypotheses 2.5(b) and Proposition 5.1(d), $\kappa^{l}$ is an isomorphism. As we now show, these two group actions are closely related. Let's write

$$
\left.P_{\pi}^{l}:=\left\langle\rho^{l}\left(G_{\pi}^{r}\right), \kappa^{l}\left(G_{\pi}^{l}\right)\right\rangle=\left\langle\rho^{l}\left(G_{\pi}^{r}\right), K_{\pi}^{l}\right)\right\rangle \subseteq \operatorname{Aut}\left(A_{0}^{l}\right) ;
$$

notice that we can (and will) mimic this set-up, starting instead from $A_{0}^{r}$ and obtaining a subgroup

$$
\left.P_{\pi}^{r}:=\left\langle\rho^{r}\left(G_{\pi}^{l}\right), \kappa^{r}\left(G_{\pi}^{r}\right)\right\rangle=\left\langle\rho^{r}\left(G_{\pi}^{l}\right), K_{\pi}^{r}\right)\right\rangle
$$

of $\operatorname{Aut}\left(A_{0}^{r}\right)$.

Lemma. Assume Hypotheses 2.5 $(a, b, c, d)$. Then $Z(A) \subseteq A_{0}$.

Proof. By Hypotheses 2.5(b), PI-deg $(A)=n$, and so, by Proposition 5.1 (d), $Z(A) \subset$ $A_{0}^{l}$. Similarly, $Z(A) \subset A_{0}^{r}$. Since $A_{0}=A_{0}^{l} \cap A_{0}^{r}$, the assertion follows.

Proposition. Assume Hypotheses 2.5 (a,b,c,d). Retain the notation stated so far in 9.2 .

(a) $P_{\pi}^{l}$ is abelian, and in fact $P_{\pi}^{l}=K_{\pi}^{l}$.

(b) The following statements are equivalent:

(1) $G_{\pi}^{l} \cap G_{\pi}^{r}=\{1\}$.

(2) $\left|G_{\pi}\right|=n^{2}$.

(3) $\rho^{l}\left(G_{\pi}^{r}\right)=K_{\pi}^{l}=P_{\pi}^{l}$.

(4) $Z(A)=A_{0}$.

(5) $\rho^{r}\left(G_{\pi}^{l}\right)=K_{\pi}^{r}=P_{\pi}^{r}$.

Proof. (a) Let $\chi \in \widehat{G_{\pi}^{l}}$. Then for all $a \in A_{0}^{l}$ and all $x \in A_{\chi}^{l} \backslash\{0\}$,

$$
x a=\kappa_{\chi}(a) x .
$$

Since $G_{\pi}^{l}$ commutes with $G_{\pi}^{r}$, $A_{\chi}^{l}$ is $G_{\pi}^{r}$-stable. So we may take $x$ in (5.2.1) to be an eigenvector for $G_{\pi}^{r}$, say $g(x)=\eta(g) x$ for all $g \in G_{\pi}^{r}$. Applying $g$ to (5.2.1) gives

$$
\eta(g) x g(a)=g\left(\kappa_{\chi}(a)\right) \eta(g) x
$$

for all $a \in A_{0}^{l}$. This proves that $g \kappa_{\chi}=\kappa_{\chi} g$, and the first claim follows.

Suppose now for a contradiction that $K_{\pi}^{l} \varsubsetneqq P_{\pi}^{l}$. Since $n$ is a unit in $k$, so is $\left|P_{\pi}^{l}\right|$ by the first claim, and so

$$
Q_{0}^{l}=\oplus_{\eta \in \widehat{P_{\pi}}}\left(Q_{0}^{l}\right)_{\eta}
$$

with each term $\left(Q_{0}^{l}\right)_{\eta} \neq 0$ by Lemma 2.2. Now $Z(A) \subseteq A_{0} \subseteq Q_{0}^{l}$ by the above lemma; moreover $Z(A)$ is fixed by the actions of $G_{\pi}^{r}$ and $K_{\pi}^{l}$ on $Q_{0}^{l}$, the former because $Z(A) \subseteq A_{0}^{r}=A^{G_{\pi}^{r}}$, the latter because $K_{\pi}^{l}$ acts by inner automorphisms of $Q(A)$. Thus, with respect to the decomposition (5.2.2), $Z(A) \subseteq\left(Q_{0}^{l}\right)_{0}$. So $\operatorname{rank}_{Z(A)}\left(A_{0}^{l}\right)>n$ and therefore $\operatorname{rank}_{Z(A)}(A)>n^{2}$, contradicting the fact that PI-deg $(A)=n$. Therefore $P_{\pi}^{l}=K_{\pi}^{l}$.

(b) (1) $\Leftrightarrow(2)$ : This is clear from the definition of $G_{\pi}$.

(2) $\Rightarrow(4)$ : Suppose that $\left|G_{\pi}\right|=n^{2}$, so that

$$
G_{\pi}=G_{\pi}^{l} \times G_{\pi}^{r}
$$


with this group of order $n^{2}$ acting faithfully on $A$. Define $A_{i j}:=A_{i}^{l} \cap A_{j}^{r}$, for $0 \leq i, j \leq n-1$, so, in particular, $A_{00}=A_{0}$. Hence there is a decomposition

$$
A=\oplus_{(i, j) \in \widehat{G_{\pi}}} A_{i j}
$$

Since (5.2.3) is a refinement of the strong grading $A=\bigoplus_{i=0}^{n-1} A_{i}^{l}$, for which the homogeneous elements are regular by Proposition 5.1 (a), $A$ is a $\widehat{G_{\pi}}$-domain. Therefore Lemma 2.2 applies, and we conclude that $A_{i j} \neq 0$ for every $(i, j) \in \widehat{G_{\pi}}$. Therefore $A_{i j}$ is a torsion free $Z(A)$-module of rank at least one. However from Hypotheses 2.5(b) and the definition of PI-degree, we know that $\operatorname{rank}_{Z(A)}(A)=n^{2}$. Therefore $\operatorname{rank}_{Z(A)}\left(A_{00}\right)=1$. In other words, $A_{00} Q(Z(A))=Q(Z(A))$, so $A_{00}$ is central and the inclusion of Lemma 5.2 is an equality.

$(4) \Rightarrow(3)$ : Suppose that $Z(A)=A_{0}=\left(A_{0}^{l}\right)^{G_{\pi}^{r}}$, so that $Z(Q)=Q_{0}=\left(Q_{0}^{l}\right)^{\rho^{l}\left(G_{\pi}^{r}\right)}$. Now the $\widehat{G_{\pi}^{r}}$-grading of $Q$ is strong, by Proposition 2.2 - equivalently, the nonzero elements of each homogeneous component are units in $Q$. Using this equivalent formulation it is immediately clear that $Q_{0}^{l}$ is strongly $\rho^{l}\left(G_{\pi}^{r}\right)$-graded, so that

$$
\operatorname{dim}_{Q_{0}}\left(Q_{0}^{l}\right)=\left|\rho\left(G_{\pi}^{r}\right)\right| .
$$

However, since PI-deg $(A)=n$ by Hypotheses 2.5(b),

$$
n^{2}=\operatorname{dim}_{Z(Q)}(Q)=\operatorname{dim}_{Q_{0}^{l}}(Q)\left(\operatorname{dim}_{Z(Q)}\left(Q_{0}^{l}\right)\right)=n \cdot \operatorname{dim}_{Z(Q)}\left(Q_{0}^{l}\right)=n \cdot \operatorname{dim}_{Q_{0}}\left(Q_{0}^{l}\right) .
$$

Hence $\operatorname{dim}_{Q_{0}}\left(Q_{0}^{l}\right)=n$, so (5.2.4) shows that $\rho^{l}$ is a monomorphism, as required.

$(3) \Rightarrow(1)$ : Suppose that $\rho^{l}\left(G_{\pi}^{r}\right)=K_{\pi}^{l}$. Recall that $K_{\pi}^{l}$ acts faithfully on $Q_{0}^{l}$, by Proposition 5.1(d). Thus our hypothesis says that $G_{\pi}^{r}$ is acting faithfully on $A_{0}^{l}=A^{G_{\pi}^{l}}$. It follows that $G_{\pi}^{l} \cap G_{\pi}^{r}=\{1\}$.

$(4) \Leftrightarrow(5)$ : This follows from the equivalence of (4) and (3), since (4) is left-right invariant.

Recall that $\widetilde{A_{0}}=\widetilde{A_{\pi}}$ is defined in Proposition $2.1(\mathrm{~g})$.

Theorem. Assume Hypotheses [2.5 $(a, b, c, d)$. Suppose that the algebra homomorphism $\pi$ in Hypotheses 2.5 satisfies the condition $G_{\pi}^{l} \cap G_{\pi}^{r}=\{1\}$. Then $A_{0}=\widetilde{A_{0}}$ that is, $A_{0}=Z(A)$ is a Hopf subalgebra of $A$.

Proof. By Proposition 5.2 (b) above, $Z(A)=A_{0}$. It remains to show that $A_{0}$ is a Hopf subalgebra of $A$. Let $a \in Z(A)$. Since $S\left(A_{0}\right)=A_{0}$ by Proposition 2.1(e), we need only to show that

$$
\Delta(a):=\sum a_{1} \otimes a_{2} \in Z(A) \otimes Z(A) .
$$

There is a decomposition

$$
A=\oplus_{(\chi, \eta) \in \widehat{G_{\pi}}} A_{\chi \eta}
$$

where $A_{\chi \eta}=A_{\chi}^{l} \cap A_{\eta}^{r}$. The argument to prove (2) $\Rightarrow$ (4) of Proposition 5.2(b) shows that

$$
A_{\chi \eta} \neq 0 \quad \forall \chi \in \widehat{G_{\pi}^{l}}, \eta \in \widehat{G_{\pi}^{r}} .
$$

Now fix $\eta \in \widehat{G_{\pi}^{r}}$, and let $0 \neq x \in A_{0, \eta}$. By Proposition 2.1(c),

$$
\Delta(x) \in A_{0}^{l} \otimes A_{\eta}^{r} \subseteq Q_{0}^{l} \otimes Q_{\eta}^{r} .
$$

For all $x^{\prime} \in A_{\eta}^{r}$ and $a^{\prime} \in A_{0}^{r}$, the definition of $\kappa_{\eta}^{r}$ in Proposition 5.1(b) yields

$$
x^{\prime} a^{\prime}=\kappa_{\eta}^{r}\left(a^{\prime}\right) x^{\prime} .
$$


The left hand entries of $\Delta(a)$ and of $\Delta(x)$ are in the commutative ring $A_{0}^{l}$, and so commute. Applying also (5.2.7) to the right hand entries of $\Delta(x)$, we find that

$$
\Delta(x) \Delta(a)=\left(I d \otimes \kappa_{\eta}^{r}\right)(\Delta(a)) \Delta(x) .
$$

Moreover, $a \in Z(A)$ and $\Delta$ is an algebra homomorphism, so that

$$
\Delta(a) \Delta(x)=\Delta(x) \Delta(a)
$$

Next, we claim that

$$
\Delta(x) \text { is a not a zerodivisor in } A \otimes A \text {. }
$$

In view of (5.2.6), it suffices to show that $Q_{\chi}^{l} \otimes Q_{\eta}^{r} \backslash\{0\}$ consists of nonzerodivisors in $Q \otimes Q$. As in the proof of Proposition 5.1 $Q_{\chi}^{l}=Q_{0}^{l} u_{\chi}$ and $Q_{\eta}^{r}=Q_{0}^{r} u_{\eta}$ with $u_{\chi}$ and $u_{\eta}$ invertible in $Q$, so it is only necessary to show that the elements of $Q_{0}^{l} \otimes Q_{0}^{r} \backslash\{0\}$ are not zero divisors in $Q \otimes Q$. But $Q_{0}^{l} \otimes Q_{0}^{r}$ is a homogeneous component of the strongly $\widehat{G_{\pi}^{l}} \times \widehat{G_{\pi}^{r}}$-graded algebra $Q \otimes Q$, whose homogeneous components have the form $Q_{\alpha}^{l} \otimes Q_{\beta}^{r}=\left(Q_{0}^{l} \otimes Q_{0}^{r}\right)\left(u_{\alpha} \otimes u_{\beta}\right)$, with $u_{\alpha} \otimes u_{\beta}$ a unit. Thus it suffices to show that $Q_{0}^{l} \otimes Q_{0}^{r}$ is a commutative domain, which follows from the fact that $k$ is algebraically closed, $[\mathrm{V}]$. This proves (5.2.10). With (5.2.8) and (5.2.9), this implies that

$$
\Delta(a)=\left(I d \otimes \kappa_{\eta}^{r}\right)(\Delta(a)) .
$$

Now all the $a_{2}$ terms in (5.2.5) are in $A_{0}^{r}$ by Proposition 2.1(c). Thus it follows from (5.2.11) that each $a_{2}$ belongs to $\left(A_{0}^{r}\right)^{\kappa_{\eta}^{r}}$. Allowing $\eta$ to vary through $\widehat{G_{\pi}^{r}}$, we see from (5.1.3) in Proposition 5.1(d) that all the terms $a_{2}$ in (5.2.5) belong to $Z(A)$. By symmetry, all the $a_{1}$ terms are in $Z(A)$ also. This completes the proof.

\section{Classification II: $i m(H)=i o(H)>1$}

6.1. Hypotheses and the main theorem. We return in $\sqrt{6}$ to the main focus of this paper, namely the structure of an affine prime regular Hopf algebra $H$ of GK-dimension one, over the algebraically closed field $k$. Recall the definitions

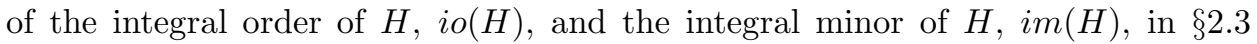
and 2.4. As noted in Theorem 2.5, $i o(H)$ equals the PI degree of $H$, and so in particular is finite, and $i m(H)$ is a positive integer dividing $i o(H)$. As in $\$ 4.1$ it will be convenient for later steps in the proof to weaken our hypotheses slightly; namely, we'll assume Hypotheses 2.5. with the homomorphism $\pi$ of Hypotheses 2.5(b) satisfying the condition:

$$
n:=\left|G_{\pi}^{l}\right|>1 \quad \text { and } \quad G_{\pi}^{l} \cap G_{\pi}^{r}=\{1\} .
$$

The main result of this section, which we now state, shows that (6.1.1) is equivalent to $i o(H)=i m(H)$ :

Theorem. Suppose $(H, \pi)$ satisfies Hypotheses 2.5 and (6.1.1). Then $H$ is isomorphic as a Hopf algebra either to the Taft algebra $H(n, t, \xi)$ of Example 3.3 with $\operatorname{gcd}(t, n)=1$, or the algebra $B(n, w, \xi)$ in Example 3.4. As a consequence, $i o(H)=i m(H)$. 
6.2. Generators and relations. Fix a primitive $n$th $\operatorname{root} \zeta$ of 1 in $k$, and define $\chi \in \widehat{G_{\pi}^{l}}$ and $\eta \in \widehat{G_{\pi}^{r}}$ by setting

$$
\chi\left(\Xi_{\pi}^{l}\right):=\zeta \text { and } \eta\left(\Xi_{\pi}^{r}\right)=\zeta .
$$

Thus $\widehat{G_{\pi}^{l}}=\left\{\chi^{i} \mid 0 \leq i \leq n-1\right\}, \widehat{G_{\pi}^{r}}=\left\{\eta^{i} \mid 0 \leq i \leq n-1\right\}$, and $\widehat{G_{\pi}}=\left\{\chi^{i} \eta^{j} \mid 0 \leq\right.$ $i, j \leq n-1\}$, so there are group gradings

$$
H=\oplus_{\chi^{i} \in \widehat{G_{\pi}^{l}}} H_{i}^{l}=\oplus_{\eta^{j} \in \widehat{G_{\pi}^{r}}} H_{j}^{r}=\oplus_{0 \leq i, j \leq n-1} H_{i j},
$$

where $H_{i j}=H_{i}^{l} \cap H_{j}^{r}$. The first two of these are strong gradings by Proposition 2.2. Since we are assuming (6.1.1), Proposition 5.2(b) and Theorem 5.2 confirm that

$$
Z(H)=H_{0}=\widetilde{H_{0}}=H_{00}
$$

In particular, this is a Hopf subalgebra of $H$, and hence is either $k[x]$ or $k\left[x^{ \pm 1}\right]$, yielding the dichotomy - primitive or group-like - of Definition 4.1 .

Note that the discussion in the proof of $(2) \Rightarrow(4)$ of Proposition 5.2(b) applies: Each $H_{i j}$ is a torsion-free - and hence free - $H_{0}$-module of rank 1 . Fix a generator $u_{i j}$ of this free module, for $i, j=0, \ldots, n-1$, with $u_{00}=1$. Then part of (6.2.1) can be rewritten as

$$
H=\oplus_{0 \leq i, j \leq n-1} H_{0} u_{i j}=\oplus_{0 \leq i, j \leq n-1} u_{i j} H_{0} .
$$

The definitions ensure that

$$
\Xi_{\pi}^{l}\left(u_{i j} a\right)=\zeta^{i} u_{i j} a \quad \text { and } \quad \Xi_{\pi}^{r}\left(u_{i j} a\right)=\zeta^{j} u_{i j} a
$$

for all $a \in H_{0}$.

Next we refine the notation for the elements of the groups $K_{\pi}^{l}$ and $K_{\pi}^{r}$ of (5.2). Fix a nonzero element $x \in H_{1}^{l}$, and define $\kappa_{l}$ to be the automorphism of $H_{0}^{l}$ defined by

$$
\kappa_{l}(a)=x a x^{-1}, \text { for all } a \in H_{0}^{l} .
$$

In view of Proposition (5.1) (b), for $i=1, \ldots, n-1$ and for $j=0, \ldots, n-1$,

$$
\kappa_{l}^{i}(a)=u_{i j} a u_{i j}^{-1} \quad \text { for all } \quad a \in H_{0}^{l} .
$$

Similarly, choosing any non-zero element $t$ of $H_{1}^{r}, \kappa_{r}$ is the automorphism of $H_{0}^{r}$ defined by $\kappa_{r}(b)=t b t^{-1}$ for all $b \in H_{0}^{r}$. Thus for $i=0, \ldots, n-1$ and for $j=$ $1, \ldots, n-1$,

$$
\kappa_{r}^{j}(b)=u_{i j} b u_{i j}^{-1} \quad \text { for all } \quad b \in H_{0}^{r} .
$$

Set $K_{\pi}^{l}=\left\langle\kappa_{l}^{1}\right\rangle=\left\{\kappa_{l}^{i}: 0 \leq i \leq n-1\right\}$ and $K_{\pi}^{r}=\left\langle\kappa_{r}^{1}\right\rangle=\left\{\kappa_{r}^{j}: 0 \leq j \leq n-1\right\}$, subgroups of $\operatorname{Aut}\left(H_{0}^{l}\right)$ and $\operatorname{Aut}\left(H_{0}^{r}\right)$ respectively. By Proposition [5.2(b) $K_{\pi}^{l}=$ $\rho^{l}\left(G_{\pi}^{r}\right)$ and $K_{\pi}^{r}=\rho^{r}\left(G_{\pi}^{l}\right)$, (where here, of course, we are carrying over the notation $\rho^{l}, \rho^{r}$ from 95.2$)$. This implies that each $H_{0 j}$ (and each $Q_{0 j}$ ) is a $K_{\pi}^{l}$-eigenspace. Thus there are $n$th roots of unity in $k, \xi_{l}$ and $\xi_{r}$ say, such that

$$
\kappa_{l}^{1}\left(u_{01}\right)=\xi_{l} u_{01}, \quad \text { and } \quad \kappa_{r}^{1}\left(u_{10}\right)=\xi_{r} u_{10} .
$$

Since the actions of $K_{\pi}^{l}$ and $K_{\pi}^{r}$ on $H_{0}^{l}$ and $H_{0}^{r}$ are faithful, by Proposition 5.1(d), $\xi_{l}$ and $\xi_{r}$ are primitive $n$th roots of unity. Using (6.2.5) and (6.2.4),

$$
u_{01} u_{10}=\kappa_{r}^{1}\left(u_{10}\right) u_{01}=\xi_{r} u_{10} u_{01}=\xi_{r} \kappa_{l}^{1}\left(u_{01}\right) u_{10}=\xi_{r} \xi_{l} u_{01} u_{10} .
$$


Thus $\xi_{r}=\xi_{l}^{-1}$. We set $\xi:=\xi_{r}$. As $\xi$ and $\zeta$ are primitive $n$th root of unity in $k$, there are integers $f$ and $f^{\prime}$, coprime to $n$ and with $1 \leq f, f^{\prime} \leq n-1$, such that $\xi=\zeta^{f}$ and $\zeta=\xi^{f^{\prime}}$.

Lemma. Retain all the above hypotheses and notation. Let $i, i^{\prime}, j$ and $j^{\prime}$ be integers from $\{0,1, \ldots, n-1\}$. Then

$$
u_{i j} u_{i^{\prime} j^{\prime}}=\xi^{i^{\prime} j-i j^{\prime}} u_{i^{\prime} j^{\prime}} u_{i j} .
$$

In particular, $H_{i j}$ commutes with $H_{i^{\prime} j^{\prime}}$ if and only if $i^{\prime} j-i j^{\prime} \equiv 0 \bmod n$.

Proof. We can pass to the simple Artinian quotient ring $Q(H)$ of $H$ by inverting $Z(H) \backslash\{0\}=H_{00} \backslash\{0\}$. In this way we see that $Q(H)=\oplus Q_{i j}$ is $\widehat{G_{\pi}}$-graded . Here, $\widehat{G_{\pi}}=\widehat{G_{\pi}^{l}} \times \widehat{G_{\pi}^{r}} \cong \mathbb{Z}_{n} \times \mathbb{Z}_{n}$, and this a strong grading, since each $Q_{i j}$ is a one-dimensional vector space over the field $Q_{0}=Q(Z(H))$, spanned by the unit $u_{i j}$ of $Q(H)$. Working inside $Q(H)$ and multiplying our original choices of the elements $u_{i j}$ by non-zero central elements as necessary, we may assume for the purposes of the present calculation that $u_{i j}=u_{10}^{i} u_{01}^{j}$. Since the adjustments are central, this will not affect the truth or otherwise of the commutation relations we are trying to prove. Now

$$
\begin{aligned}
u_{i j} u_{i^{\prime} j^{\prime}}\left(u_{i^{\prime} j^{\prime}} u_{i j}\right)^{-1} & =\left(u_{10}\right)^{i}\left(u_{01}\right)^{j}\left(u_{10}\right)^{i^{\prime}}\left(u_{01}\right)^{j^{\prime}}\left(u_{01}\right)^{-j}\left(u_{10}\right)^{-i}\left(u_{01}\right)^{-j^{\prime}}\left(u_{10}\right)^{-i^{\prime}} \\
& =\xi_{r}^{i^{\prime} j-i j^{\prime}}=\xi^{i^{\prime} j-i j^{\prime}}
\end{aligned}
$$

as required.

Proposition. Retain the above hypotheses and notation. Let $i, j \in\{0,1, \ldots, n-1\}$.

(a) Conjugation of $Q(H)$ by $u_{i j}$ restricts to an automorphism of $H$, namely $\left(\left(\Xi_{\pi}^{l}\right)^{j}\left(\Xi_{\pi}^{r}\right)^{-i}\right)^{f}$. That is,

$$
\left(\left(\Xi_{\pi}^{l}\right)^{j}\left(\Xi_{\pi}^{r}\right)^{-i}\right)^{f}(x)=u_{i j} x u_{i j}^{-1}
$$

for all $x \in H$.

(b) $\left(\Xi_{\pi}^{l}\right)^{f}(x)=u_{01} x u_{01}^{-1}$ and $\left(\Xi_{\pi}^{r}\right)^{f}=u_{10}^{-1} x u_{10}$ for all $x \in H$.

(c) The element $u_{i j}$ is normal in $H$.

Proof. (a) By (6.2.3), $H=\oplus_{i j} u_{i j} H_{0}$ and the center $H_{0}$ is fixed by $\Xi_{l}$ and $\Xi_{r}$. It suffices therefore to show that

$$
\left(\left(\Xi_{\pi}^{l}\right)^{j}\left(\Xi_{\pi}^{r}\right)^{-i}\right)^{f}\left(u_{i^{\prime} j^{\prime}}\right)=u_{i j} u_{i^{\prime} j^{\prime}} u_{i j}^{-1}
$$

This follows from the above lemma.

$(\mathrm{b}, \mathrm{c})$ are special cases of (a).

6.3. The ideal $J_{i q}$. Recall the definition in (2.3) of the integral annihilator $J_{i q}$, and recall the comment which follows Hypotheses 2.5 .

Lemma. Continue with the notations and hypotheses introduced so far in $\S 6$. Let $i, j \in\{0,1, \ldots, n-1\}$,

(a) $S\left(H_{i}^{l}\right)=H_{-i}^{r}$ and $S\left(H_{i j}\right)=H_{-j,-i}$, (where the suffixes are interpreted $\bmod n)$.

(b) If $i \neq j$ then $\epsilon\left(H_{i j}\right)=0$.

(c) $\epsilon\left(u_{i i}\right) \neq 0$.

(d) If $i \neq j$ then $H_{i j} \subseteq J_{i q}$. 
Proof. (a) By Proposition 2.1(e), $S\left(\Xi_{\pi}^{l}\right)^{-1}=\Xi_{\pi}^{r} S$, so that $\left(\Xi_{\pi}^{r}\right)^{-1} S=S \Xi_{\pi}^{l}$. Bearing in mind that $S$ is injective, this implies that $S\left(H_{i}^{l}\right)=H_{-i}^{r}$. The second claim follows from the first, since $H_{i j}=H_{i}^{l} \cap H_{j}^{r}$.

(b) Let $i, j \in\{0,1, \ldots, n-1\}$ and let $x \in H_{i j}$. By Proposition 2.2(a) and its right-hand version, $\Delta(x) \in H_{i}^{l} \otimes H_{j}^{r}$. Therefore, using (a),

$$
\epsilon(x)=m_{H} \circ(\operatorname{Id} \otimes S) \circ \Delta(x) \in H_{i}^{l} H_{-j}^{l}=H_{i-j}^{l} .
$$

Thus, if $i \neq j, \epsilon(x) \in H_{0}^{l} \cap H_{i-j}^{l}=\{0\}$.

(c) Suppose that $i=j$. By (b) and Proposition 2.2(a),

(6.3.1) $u_{i i}=m_{H} \circ(\epsilon \otimes \mathrm{Id}) \circ \Delta\left(u_{i i}\right) \in m_{H} \circ(\epsilon \otimes \mathrm{Id})\left(H_{i}^{l} \otimes H_{i}^{r}\right)=\epsilon\left(H_{i}^{l}\right) H_{i}^{r}$.

Since $H_{i}^{l}=\oplus_{j} H_{i j}$ it follows from (6.3.1) and (b) that $\epsilon\left(H_{i i}\right) \neq 0$. But $H_{i i}=u_{i i} H_{0}$, so $\epsilon\left(u_{i i}\right) \neq 0$.

(d) By (b), $H_{i j} \subseteq$ ker $\epsilon$. But $H_{i j}$ is $G_{\pi}^{l}$-invariant, so it follows that

$$
H_{i j} \subseteq \cap_{i \geq 0}\left(\Xi_{\pi}^{l}\right)^{i}(\operatorname{ker} \epsilon)=J_{i q},
$$

the equality being Theorem $2.3(\mathrm{e})$.

6.4. The twistor. We shall approach the coalgebra and algebra structure of the prime Hopf algebra $H$ of GK-dimension one by first studying a finite dimensional Hopf factor of $H$, as follows. By Theorem $5.2 H_{0}$ is a central Hopf subalgebra of $H$. Hence there is an exact sequence of Hopf algebras

$$
0 \rightarrow H_{0} \rightarrow H \rightarrow H_{t w} \rightarrow 0,
$$

where $H_{t w}$ is the Hopf algebra $H /\left(\operatorname{ker} \epsilon \cap H_{0}\right) H$. We call $H_{t w}$ the twistor of $H$. Recall that, by (6.2.3), $H$ is a free $H_{0}$-module of rank $n^{2}$ on the basis $\left\{u_{i j}\right\}$. Clearly, $\operatorname{dim}_{k} H_{t w}=n^{2}$, with a basis $\left\{v_{i j}\right\}_{0 \leq i, j \leq n-1}$ where $v_{i j}$ is the image in $H_{t w}$ of the element $u_{i j}$ for every pair $(i, j)$. It will be convenient also to denote the ideal (ker $\left.\epsilon \cap H_{0}\right) H$ of $H$ by $J_{t w}$. Note that, by Proposition 2.3. $J_{t w} \subseteq J_{i q}$.

Examples. (a) Let $H=H(n, t, \xi)$ be a Taft algebra as in 33.3 , so $i o(H)=n$. By (3.3.1), $\operatorname{im}(H)=i o(H)$ if and only if $t$ is coprime to $n$. Replacing $g$ by $g^{t}$ and $\xi$ by $\xi^{\prime}:=\xi^{t}$, we may assume that $t=1$. The twistor $H(n, 1, \xi)_{t w}$ of this Hopf algebra is then

$$
H\left(n, 1, \xi^{\prime}\right)_{t w}:=k\langle g, x\rangle /\left(x^{n}=0, g^{n}=1, g x=\xi^{\prime} x g\right),
$$

with $\Delta(g)=g \otimes g$ and $\Delta(x)=x \otimes g+1 \otimes x$.

(b) Let $B=B(n, w, \xi)$ be one of the examples from $\$ 3.4$ As noted there, $B_{0}=k\left[x^{ \pm 1}\right]$, so that $B_{t w}=B /(x-1) B$ and it follows at once that $B(n, w, \xi)_{t w} \cong$ $H(n, 1, \xi)_{t w}$.

6.5. Coalgebra structure of $H_{t w}$. The key to the coalgebra structure rests in the comultiplication rules for the basis elements $v_{i j}$. Now $v_{00}=1$ (because $u_{00}=1$ ) and, thanks to Lemma 6.3 , after adjustment by suitable non-zero scalars we can assume that, in $H$,

$$
\epsilon\left(u_{i j}\right)=\delta_{i j}
$$

so that, in $H_{t w}$,

$$
\epsilon\left(v_{i j}\right)=\delta_{i j}
$$

for all $i, j=0,1, \ldots, n-1$. 
Lemma. Continue with the notations and hypotheses of $\S 6$. The comultiplication $\Delta$ in $H_{t w}$ satisfies the following properties.

(a) For $i=0, \ldots, n-1$,

$$
\Delta\left(v_{i i}\right)=v_{i i} \otimes v_{i i}+\sum_{s \neq i} c_{s s}^{i i} v_{i s} \otimes v_{s i},
$$

with $c_{s s}^{i i}=c_{i i}^{s s}$ for all $i \neq s$.

(b) For $i, j=0, \ldots, n-1$, with $i \neq j$,

$$
\Delta\left(v_{i j}\right)=v_{i i} \otimes v_{i j}+v_{i j} \otimes v_{j j}+\sum_{s \neq i, j} c_{s s}^{i j} v_{i s} \otimes v_{s j}
$$

for scalars $c_{s s}^{i j}$ satisfying the relations

$$
c_{t t}^{i j} c_{s s}^{i t}=c_{s s}^{i j} c_{t t}^{s j}
$$

for all mutually distinct $i, j, s, t$ in $\{0, \ldots, n-1\}$.

(c) For all mutually distinct $i, s, t$ in $\{0, \ldots, n-1\}$,

$$
c_{s s}^{t t}=c_{s s}^{i t} c_{t t}^{i s}=c_{s s}^{t i} c_{t t}^{s i} .
$$

Proof. First, by Proposition 2.2 (a) and the fact that $u_{i j} \in H_{i j}=H_{i}^{l} \cap H_{j}^{r}$, there are elements $c_{s t}^{i j}$ of $k$ such that for all $i, j=0, \ldots, n-1$,

$$
\Delta\left(v_{i j}\right)=\sum_{s, t} c_{s t}^{i j} v_{i s} \otimes v_{t j} .
$$

Fix $i$, consider (6.5.3) with $j=i$, and apply $m_{H} \circ(\operatorname{Id} \otimes \epsilon)$ and $m_{H} \circ(\epsilon \otimes \operatorname{Id})$ to the right hand side. Since the outcome in both cases is $v_{i i}$ we deduce from (6.5.2) and the linear independence over $k$ of the $v_{i j}$ that

$$
\Delta\left(v_{i i}\right)=v_{i i} \otimes v_{i i}+\sum_{s, t \neq i} c_{s t}^{i i} v_{i s} \otimes v_{t i} .
$$

Similarly we have, for $i \neq j$,

$$
\Delta\left(v_{i j}\right)=v_{i i} \otimes v_{i j}+v_{i j} \otimes v_{j j}+\sum_{s \neq i, t \neq j} c_{s t}^{i j} v_{i s} \otimes v_{t j} .
$$

Applying $(\operatorname{Id} \otimes \Delta)$ and $(\Delta \otimes \operatorname{Id})$ to (6.5.4) shows that

$$
\begin{aligned}
& (\operatorname{Id} \otimes \Delta) \circ \Delta\left(v_{i i}\right)=v_{i i} \otimes v_{i i} \otimes v_{i i}+\sum_{l, p \neq i} c_{l p}^{i i}\left(v_{i i} \otimes v_{i l} \otimes v_{p i}\right)+ \\
& \sum_{s, t \neq i} c_{s t}^{i i} v_{i s} \otimes\left(v_{t t} \otimes v_{t i}+v_{t i} \otimes v_{i i}\right)+\sum_{l \neq t, p \neq i} \sum_{s, t \neq i} c_{l p}^{t i} c_{s t}^{i i}\left(v_{i s} \otimes v_{t l} \otimes v_{p i}\right),
\end{aligned}
$$

and

$$
\begin{aligned}
& (\Delta \otimes \mathrm{Id}) \circ \Delta\left(v_{i i}\right)=v_{i i} \otimes v_{i i} \otimes v_{i i}+\sum_{w, u \neq i} c_{w u}^{i i}\left(v_{i w} \otimes v_{u i} \otimes v_{i i}\right)+ \\
& \sum_{s, t \neq i} c_{s t}^{i i}\left(v_{i s} \otimes v_{s s}+v_{i i} \otimes v_{i s}\right) \otimes v_{t i}+\sum_{w \neq i, u \neq s} \sum_{s, t \neq i} c_{w u}^{i s} c_{s t}^{i i}\left(v_{i w} \otimes v_{u s} \otimes v_{t i}\right),
\end{aligned}
$$

respectively. Using the above expressions and canceling out the equal terms from the left-hand and right-hand sides of the equation

$$
(\mathrm{Id} \otimes \Delta) \circ \Delta\left(v_{i i}\right)=(\Delta \otimes \mathrm{Id}) \circ \Delta\left(v_{i i}\right)
$$


we obtain

$$
\begin{gathered}
\sum_{s, t \neq i} c_{s t}^{i i} v_{i s} \otimes v_{t t} \otimes v_{t i}+\sum_{l \neq t, p \neq i} \sum_{s, t \neq i} c_{l p}^{t i} c_{s t}^{i i} v_{i s} \otimes v_{t l} \otimes v_{p i}= \\
\sum_{s, t \neq i} c_{s t}^{i i} v_{i s} \otimes v_{s s} \otimes v_{t i}+\sum_{w \neq i, u \neq s} \sum_{s, t \neq i} c_{w u}^{i s} c_{s t}^{i i} v_{i w} \otimes v_{u s} \otimes v_{t i} .
\end{gathered}
$$

Comparing the terms with $v_{i s} \otimes v_{t t} \otimes v_{t i}$, it follows that

$$
c_{s t}^{i i}=0
$$

for all $s \neq t$. Thus we have proved the first part of (a).

Substituting (6.5.7) into (6.5.6) and re-arranging the indices we have

$$
\sum_{l \neq w, p \neq i} \sum_{w \neq i} c_{l p}^{w i} c_{w w}^{i i} v_{i w} \otimes v_{w l} \otimes v_{p i}=\sum_{w \neq i, u \neq p} \sum_{p \neq i} c_{w u}^{i p} c_{p p}^{i i} v_{i w} \otimes v_{u p} \otimes v_{p i} .
$$

This implies that

$$
\begin{cases}c_{l p}^{w i} c_{w w}^{i i}=0 & p \neq l \\ c_{w u}^{i p} c_{p p}^{i i}=0 & w \neq u \\ c_{p p}^{w i} c_{w w}^{i i}=c_{w w}^{i p} c_{p p}^{i i} . & \end{cases}
$$

In proving (b) and (c), let's look first at the case when $n=2$. Since $v_{00}=1$, $c_{11}^{00}=0$. If $c_{00}^{11}$ is nonzero, then $c_{w u}^{01}=c_{w u}^{10}=0$ for $w \neq u$ by the first two of the above three equations, and so 6.5.5 becomes

$$
\Delta\left(v_{i j}\right)=v_{i i} \otimes v_{i j}+v_{i j} \otimes v_{j j}
$$

for $i \neq j$. By comparing $(\operatorname{Id} \otimes \Delta) \circ \Delta\left(v_{01}\right)$ with $(\Delta \otimes \operatorname{Id}) \circ \Delta\left(v_{01}\right)$ we obtain that $c_{00}^{11}=c_{11}^{00}=0$, a contradiction. Hence in this case, thanks to (a), $v_{11}$ is a group-like element. By (6.5.5), we have

$$
\Delta\left(v_{01}\right)=v_{00} \otimes v_{01}+v_{01} \otimes v_{11}+c_{10}^{01} v_{01} \otimes v_{01}
$$

By comparing $(\mathrm{Id} \otimes \Delta) \circ \Delta\left(v_{01}\right)$ with $(\Delta \otimes \mathrm{Id}) \circ \Delta\left(v_{01}\right)$ again we obtain $c_{10}^{01}=0$. Similarly, $c_{01}^{10}=0$. Thus, in view of (a) and these observations, we have proved that $v_{11}$ is a group-like element and that both $v_{01}$ and $v_{10}$ are skew primitive elements. Therefore (a), (b) and (c) hold in the case $n=2$.

We return now to the case where $n>2$. Fix $i$ and $j$ with $i \neq j$. We proceed as in the case $i=j$, between (6.5.5) and (6.5.7); since the arguments are similar we give fewer details. Calculating the left-hand and the right-hand sides of the equation

$$
(\mathrm{Id} \otimes \Delta) \circ \Delta\left(v_{i j}\right)=(\Delta \otimes \mathrm{Id}) \circ \Delta\left(v_{i j}\right),
$$

and canceling equal terms (where we omit the detailed expressions), we find that

$$
\begin{aligned}
& \sum_{s \neq j} c_{s s}^{j j} v_{i j} \otimes v_{j s} \otimes v_{s j}+\sum_{s \neq i, t \neq j} c_{s t}^{i j} v_{i s} \otimes\left(v_{t t} \otimes v_{t j}+\sum_{w \neq t, u \neq j} c_{w u}^{t j} v_{t w} \otimes v_{u j}\right) \\
& =\sum_{s \neq i} c_{s s}^{i i} v_{i s} \otimes v_{s i} \otimes v_{i j}+\sum_{s \neq i, t \neq j} c_{s t}^{i j}\left(v_{i s} \otimes v_{s s}+\sum_{p \neq i, l \neq s} c_{p l}^{i s} v_{i p} \otimes v_{l s}\right) \otimes v_{t j} .
\end{aligned}
$$

By comparing the terms with $v_{i s} \otimes v_{t t} \otimes v_{t j}$, we see that $c_{s t}^{i j}=0$ for all $s \neq t$. Hence the first claim in (b) follows. Substituting this into (6.5.8) and deleting the equal terms on left and right, we have 


$$
\begin{aligned}
\sum_{s \neq j} c_{s s}^{j j} v_{i j} \otimes v_{j s} \otimes v_{s j}+\sum_{s \neq i, j} c_{s s}^{i j} v_{i s} \otimes\left(\sum_{w \neq s, j} c_{w w}^{s j} v_{s w} \otimes v_{w j}\right) \\
=\sum_{s \neq i} c_{s s}^{i i} v_{i s} \otimes v_{s i} \otimes v_{i j}+\sum_{s \neq i, j} c_{s s}^{i j}\left(\sum_{p \neq i, s} c_{p p}^{i s} v_{i p} \otimes v_{p s} \otimes v_{s j}\right) .
\end{aligned}
$$

Comparing the term $v_{i j} \otimes v_{j i} \otimes v_{i j}$, it follows that

$$
c_{j j}^{i i}=c_{i i}^{j j},
$$

proving the rest of (a).

Comparing terms $v_{i j} \otimes v_{j s} \otimes v_{s j}$ for $s \neq i, j$ yields

$$
c_{s s}^{j j}=c_{s s}^{i j} c_{j j}^{i s} \text {. }
$$

Comparing terms $v_{i s} \otimes v_{s i} \otimes v_{i j}$ for $s \neq i, j$ we deduce

$$
c_{s s}^{i j} c_{i i}^{s j}=c_{s s}^{i i} \text {. }
$$

Combining the last two equations yields

$$
c_{s s}^{t t}=c_{s s}^{i t} c_{t t}^{i s}=c_{s s}^{t i} c_{t t}^{s i}
$$

for all distinct $s, t, i$. That is, (c) is proved. Finally, by comparing terms $v_{i s} \otimes v_{s t} \otimes v_{t j}$ for distinct $i, j, s, t$, we obtain

$$
c_{s s}^{i j} c_{t t}^{s j}=c_{t t}^{i j} c_{s s}^{i t},
$$

which is the second part of (b). This completes the proof.

\subsection{Algebra and coalgebra structure of $H_{t w}$.}

Proposition. Keep all the notation introduced so far in $9 \sqrt{6}$. The following relations hold in $H_{t w}$.

(a) Let $g=v_{11}$. Then $g^{n}=1$ and for $i=0,1, \ldots, n-1, v_{i i}=g^{i}$.

(b) For all $i, j=0,1, \ldots, n-1$, with $i \neq j, v_{i j}^{n}=0$.

(c) For all $i, j=0,1, \ldots, n-1, g v_{i j}=\xi^{i-j} v_{i j} g$. In particular, $g v_{10}=\xi v_{10} g$ and $g v_{01}=\xi^{-1} v_{01} g$.

(d) The element $g$ is group-like in $H_{t w}$. As a consequence

$$
c_{s s}^{t t}=0 \quad \text { and } \quad c_{s s}^{i t} c_{t t}^{i s}=c_{s s}^{t i} c_{t t}^{s i}=0
$$

for all distinct $s, t, i$.

(e) We may extend the expressions for $v_{i i}$ in (a) by defining $v_{i j}:=g^{i} v_{0(j-i)}$ for all $i, j$, where $j-i$ is to be interpreted modulo $n$ when $i>j$. Once this is done, $c_{s s}^{i j}=c_{(s-t)(s-t)}^{(i-t)(j-t)}$ for all $i, j, s, t$ with $i, j$ and $s$ distinct.

(f) There exists $t$ coprime to $n, 1 \leq t \leq n-1$, such that $k\left\langle v_{0 t}\right\rangle$ contains $v_{0 i}$ for all $i=0,1, \ldots, n-1$.

(g) As an algebra, $H_{t w}$ is isomorphic to the twistor $H\left(n, 1, \xi^{t}\right)_{t w}$ of the Taft algebra, when we take the generators to be $y=v_{0 t}$ and $g=v_{t t}$.

Proof. (a) Since $H_{t w}$ is graded, $g^{n} \in k v_{00}=k$. By (6.5.2), $\epsilon\left(g^{n}\right)=1^{n}=1$. Hence $g^{n}=1$. In particular, $g$ is invertible. Both $g^{i}$ and $v_{i i}$ are nonzero elements in the 1-dimensional space $k v_{i i}$. Then (6.5.2) shows that they are equal.

(b) Let $i \neq j$. Since $H_{t w}$ is graded, $v_{i j}^{n} \in k v_{00}=k$. The assertion follows from (6.5.2).

(c) This follows at once from Lemma 6.2 
(d) By Lemma 6.5(a)

$$
\Delta\left(v_{11}\right)=v_{11} \otimes v_{11}+\sum_{s \neq i} c_{s s}^{11} v_{1 s} \otimes v_{s 1}
$$

that is,

$$
\Delta(g)=g \otimes g+\alpha
$$

where $\alpha:=\sum_{s \neq i} c_{s s}^{11} v_{1 s} \otimes v_{s 1}$ commutes with $g \otimes g$ by (c). Since $g^{n}=1, \Delta(g)^{n}=$ $1 \otimes 1$. This implies that

$$
\sum_{i=0}^{n}\left(\begin{array}{c}
n \\
i
\end{array}\right)(g \otimes g)^{n-i} \alpha^{i}=1 \otimes 1
$$

which is equivalent to

$$
\alpha\left\{n\left(g^{n-1} \otimes g^{n-1}\right)+\sum_{i=2}^{n}\left(\begin{array}{c}
n \\
i
\end{array}\right)(g \otimes g)^{n-i} \alpha^{i-1}\right\}=0 .
$$

Since $\beta:=\sum_{i=2}^{n}\left(\begin{array}{c}n \\ i\end{array}\right)(g \otimes g)^{n-i} \alpha^{i-1}$ is nilpotent by (b) and Lemma 6.2. $n\left(g^{n-1} \otimes\right.$ $\left.g^{n-1}\right)+\beta$ is invertible. Hence $\alpha=0$, and $g$ is group-like as claimed. Since $v_{i i}=v_{11}^{i}$ for $i=1, \ldots, n-1$, these elements too are group-like. Hence the remaining claims in (d) follow from Lemma 6.5(c).

(e) Since the proposed elements $v_{i j}$ are non-zero and lie in the correct subspace, the first part is clear. The second part follows by using Lemma 6.5(b) and the fact that $\Delta$ is an algebra homomorphism to expand the equation $\Delta\left(v_{i j}\right)=$ $\Delta\left(g^{t} v_{(i-t)(j-t)}\right)$.

(f) Set $B:=\left(H_{0}^{l}+J_{t w}\right) / J_{t w}$. Then $B=\oplus_{0 \leq i \leq n-1} k v_{0 i}$. By (b) and Lemma 6.2 $B$ is a local ring with Jacobson radical

$$
J=\oplus_{0<i \leq n-1} k v_{0 i}=\left(H_{0}^{l} \cap J_{i q}\right)+J_{t w} / J_{t w} .
$$

However, $B$ is a (finite dimensional) factor of $H_{0}^{l}$, which is a Dedekind domain by Hypotheses 2.5(c). Thus $B$ is a principal ideal ring, and so its radical $J$ is generated by the (unique) element $v_{0 t} \in J \backslash J^{2}$. By induction, $J^{i}=k\left(v_{0 t}\right)^{i}+J^{i+1}$ provided $J^{i} \neq 0$. Thus the $k$-subalgebra of $B$ generated by $v_{0 t}$ includes all $v_{0 i}$ and therefore equals $B$. That is, $B=k[y] /\left(y^{n}\right)$ where $y:=v_{0 t}$. It is clear from the grading that $t$ must be coprime to $n$.

(g) By (e) and (f), $H_{t w}$ is generated by $g$ and $y$. Our calculations so far show that, as an algebra, $H_{t w}$ is a factor of the algebra

$$
k\langle g, y\rangle /\left\langle y^{n}=0, g^{n}=1, g y=\xi^{-t} y g\right\rangle .
$$

But this latter algebra is isomorphic to $H\left(n, 1, \xi^{t}\right)_{t w}$, and so comparing dimensions we see that in fact the two algebras are isomorphic.

Retain all the notation and hypotheses of 96 . We can now completely describe the twistor of $H$ :

Theorem. Suppose $(H, \pi)$ satisfies Hypotheses 2.5 and (6.1.1). There exists an $n$th primitive root of $1, \xi$, such that the twistor $H_{t w}$ of $H$ is isomorphic as a Hopf algebra to the twistor $H(n, 1, \xi)_{t w}$ of the Taft algebra $H(n, 1, \xi)$, as described in Examples 6.4. The basis elements $\left\{v_{i j}: 0 \leq i, j \leq n-1\right\}$ can be chosen to be

$$
v_{i j}:= \begin{cases}g^{i} y^{j-i} & i \leq j, \\ g^{i} y^{n+j-i}, & i>j .\end{cases}
$$


Proof. Let $t$ be the integer determined by Proposition 6.6(f) and let $t^{\prime}$ be an integer such that $t^{\prime}$ is the multiplicative inverse of $t$ in $C_{n}$. We replace $\pi$ by its $t^{\prime}$ th power $\pi^{* t^{\prime}}$ in $G\left(H^{\circ}\right)$. It is clear that Hypotheses 2.5 and (6.1.1) hold for this new $\pi$. Doing so allows us to assume that $y=v_{01}$. Adjusting $g$ accordingly, we continue to take $g=v_{11}$. By Proposition $6.6(\mathrm{~g}), H_{t w} \cong H(n, 1, \xi)_{t w}$ as algebras. Since $\epsilon$ is already determined by (6.5.2), and the antipode, being the inverse of the identity map under convolution, is determined by the bialgebra structure, it remains only to compare the coalgebra structures. By Proposition[6.6(d) $\Delta(g)=g \otimes g$, and Lemma 6.5 (b) shows that

$$
\Delta(y)=1 \otimes y+y \otimes g+\sum_{s \neq 0,1} c_{s s}^{01} v_{0 s} \otimes v_{s 1}=1 \otimes y+y \otimes g+\left(y^{2} \otimes y^{2}\right) f
$$

where $f \in H_{t w} \otimes H_{t w}$. The result will follow if we can show that

$$
c_{s s}^{01}=0 \text { for all } s>1 \text {. }
$$

Using (6.6.1) and the facts that $y^{n}=0$ and $y g=\xi^{t} g y$, we calculate that, for all $1<j \leq n-1$,

$$
\Delta\left(y^{j}\right)=1 \otimes y^{j}+y^{j} \otimes g^{j}+\left(\sum_{i=1}^{j-1}\left(\begin{array}{l}
j \\
i
\end{array}\right)_{\xi^{t}} y^{i} \otimes g^{i} y^{j-i}\right)+h_{j},
$$

where the total $y$-degree of $h_{j}$ is greater than $j+1$. Since $\xi^{t}$ is a primitive $n$th root of unity, the coefficient

$$
c_{i i}^{0 j}=\left(\begin{array}{l}
j \\
i
\end{array}\right)_{\xi^{t}} \neq 0
$$

for all $1 \leq i \leq j-1$. It follows from the second equation in Proposition 6.6(d) that

$$
c_{j j}^{0 i}=0
$$

for all $0<i<j$, proving (6.6.2). It is now clear that the final adjustments to the choice of basis $\left\{v_{i j}\right\}$ can be made as proposed.

6.7. Lifting the coalgebra structure. In the previous two subsections we have shown that $v_{11}$ is group-like and $v_{01}$ is $\left(v_{11}, 1\right)$-primitive. The goal of this section is to show that $u_{11}$ is group-like and $u_{01}$ is $\left(u_{11}, 1\right)$-primitive.

Recall from 6.2 .2 that $Z(H)=H_{0}$ is either $k[x]$ or $k\left[x^{ \pm 1}\right]$. We shall use $z$ to denote $x$ in the first case and $x-1$ in the second case, so that

$$
J_{t w} \cap H_{0}=\operatorname{ker} \epsilon \cap H_{0}=\langle z\rangle .
$$

The following is clear:

Lemma. Suppose $(H, \pi)$ satisfies Hypotheses 2.5 and (6.1.1).

(a) $H / z^{n} H$ is finite dimensional for all $n$, and $\bigcap_{n \geq 0} z^{n} H=\{0\}$.

(b) $H / z H=H_{t w}$.

(c) $H_{t w} / J a c\left(H_{t w}\right)=H_{i q}=k \mathbb{Z}_{n}$ where Jac(-) is the Jacobson radical.

By Proposition 2.2(a) and the fact that $H_{0} u_{i j}=H_{i j}=H_{i}^{l} \cap H_{j}^{r}$, there are elements $C_{s t}^{i j}$ of $H_{0} \otimes H_{0}$ such that for all $i, j=0, \ldots, n-1$,

$$
\Delta\left(u_{i j}\right)=\sum_{s, t} C_{s t}^{i j} u_{i s} \otimes u_{t j}
$$


Clearly, $(\epsilon \otimes \epsilon)\left(C_{s t}^{i j}\right)=c_{s t}^{i j}$. For $C_{s t}^{i j}=\sum c_{i} \otimes d_{i} \in H_{0} \otimes H_{0}$, let's write $\left[C_{s t}^{i j}\right]_{13}$ to denote the element $\sum_{i} c_{i} \otimes 1 \otimes d_{i} \in H_{0} \otimes H_{0} \otimes H_{0}$.

Proposition. Suppose $(H, \pi)$ satisfies Hypotheses 2.5 and 6.1.1).

(a) The element $u_{11}$ of $H$ is group-like. That is, $C_{11}^{11}=1$ and $C_{s t}^{11}=0$ for all $(s, t) \neq(1,1)$. Furthermore, we may define $u_{i i}:=u_{11}^{i}$ for all $i=1, \cdots, n-1$, so that $u_{i i}$ is group-like for all $i=1, \ldots, n-1$.

(b) After a possible adjustment, $u_{01}$ is a $\left(u_{11}, 1\right)$-primitive; that is,

$$
\Delta\left(u_{01}\right)=1 \otimes u_{01}+u_{01} \otimes u_{11} .
$$

Proof. Let $C_{s t}^{i j}$ be defined as in (6.7.1). We will repeat some of the computations in (6.5) and (6.6) to prove the following preliminary steps towards (a) and (b):

$$
\left[(\Delta \otimes 1) C_{s t}^{i j}\right]\left[C_{\alpha \beta}^{i s} \otimes 1\right]=\left[(1 \otimes \Delta) C_{\alpha \beta}^{i j}\right]\left[1 \otimes C_{s t}^{\beta j}\right]
$$

for all $i, j, s, t, \alpha, \beta$;

$$
(\epsilon \otimes 1) C_{i i}^{i j}=1 \quad \text { and } \quad(1 \otimes \epsilon) C_{j j}^{i j}=1
$$

for all $i$ and $j$;

$$
(\epsilon \otimes 1) C_{s t}^{i j}=0 \quad \text { and } \quad(1 \otimes \epsilon) C_{s t}^{i j}=0
$$

for all $i, j, s, t$ with $s \neq t$;

$$
C_{s t}^{i j}=0
$$

for all $i, j, s, t$ with $s \neq t$;

$$
C_{\text {ss }}^{11}=0 \text { for all } s \neq 1 \text {, so } \Delta\left(u_{11}\right)=C_{11}^{11} u_{11} \otimes u_{11} .
$$

Proof of (6.7.2): Applying $(\Delta \otimes 1)$ and $(1 \otimes \Delta)$ to (6.7.1) we have

$$
\begin{aligned}
& (\Delta \otimes 1) \Delta\left(u_{i j}\right)=\sum\left[(\Delta \otimes 1) C_{s t}^{i j}\right]\left[C_{\alpha \beta}^{i s} \otimes 1\right] u_{i \alpha} \otimes u_{\beta s} \otimes u_{t j} \\
& (1 \otimes \Delta) \Delta\left(u_{i j}\right)=\sum\left[(1 \otimes \Delta) C_{\alpha \beta}^{i j}\right]\left[1 \otimes C_{s t}^{\beta j}\right] u_{i \alpha} \otimes u_{\beta s} \otimes u_{t j}
\end{aligned}
$$

Now (6.7.2) follows from the above equations and the fact that $(\Delta \otimes 1) \circ \Delta=$ $(1 \otimes \Delta) \circ \Delta$.

Proof of (6.7.3): By definition and Lemma6.5(a,b), $(\epsilon \otimes \epsilon)\left(C_{i i}^{i j}\right)=c_{i i}^{i j}=1$. Hence $w:=(\epsilon \otimes 1)\left(C_{i i}^{i j}\right)$ is nonzero. Applying $\epsilon \otimes \epsilon \otimes 1$ to (6.7.2) with $\alpha=\beta=s=t=i$ and using the fact that $(\epsilon \otimes 1) \circ \Delta(c)=1 \otimes c$, we obtain

$\left[(1 \otimes \epsilon \otimes 1)\left(1 \otimes C_{i i}^{i j}\right)\right]\left[\left((\epsilon \otimes \epsilon) C_{i i}^{i i}\right) \otimes 1\right]=\left[(\epsilon \otimes 1 \otimes 1)\left[C_{i i}^{i j}\right]_{13}\right]\left[(\epsilon \otimes \epsilon \otimes 1)\left(1 \otimes C_{i i}^{i j}\right)\right]$. Since $(\epsilon \otimes \epsilon) C_{i i}^{i i}=c_{i i}^{i i}=1$, the above equation reduces to

$$
1 \otimes w=(1 \otimes w)(1 \otimes w)
$$

and since $H_{0} \otimes H_{0} \otimes H_{0}$ is a domain, $w=1$. The second equation $(1 \otimes \epsilon) C_{j j}^{i j}=1$ follows by symmetry.

Proof of (6.7.4): Applying $1 \otimes \epsilon \otimes \epsilon$ to (6.7.2) with $\alpha=\beta=s \neq t$, we find that $\left[(1 \otimes 1 \otimes \epsilon)\left(\left[C_{s t}^{i j}\right]_{13}\right)\right]\left[(1 \otimes \epsilon)\left(C_{s s}^{i s}\right) \otimes 1\right]=\left[(1 \otimes \epsilon \otimes 1)\left(C_{s s}^{i j} \otimes 1\right)\right]\left[1 \otimes(\epsilon \otimes \epsilon)\left(C_{s t}^{s j}\right)\right]$.

By $\left(\underline{6.7 .3)}(1 \otimes \epsilon)\left(C_{s s}^{i s}\right)=1\right.$ and by Lemma $6.5(\mathrm{a}, \mathrm{b}),(\epsilon \otimes \epsilon)\left(C_{s t}^{s j}\right)=c_{s t}^{s j}=0$, since $s \neq t$. Therefore $(1 \otimes 1 \otimes \epsilon)\left(\left[C_{s t}^{i j}\right]_{13}\right)=0$; that is, $(1 \otimes \epsilon)\left(C_{s t}^{i j}\right)=0$ for all $i, j, s, t$ with $s \neq t$. This is the second equation in (6.7.4). The first one can be deduced similarly. 
Proof of (6.7.5): Applying $1 \otimes \epsilon \otimes 1$ to (6.7.2) with $\alpha \neq \beta=s=t$, yields

$$
\left[C_{s s}^{i j}\right]_{13}\left[(1 \otimes \epsilon)\left(C_{\alpha s}^{i s}\right) \otimes 1\right]=\left[C_{\alpha s}^{i j}\right]_{13}\left(1 \otimes(\epsilon \otimes 1)\left(C_{s s}^{s j}\right)\right) .
$$

Since $(1 \otimes \epsilon)\left(C_{\alpha s}^{i s}\right)=0$ by (6.7.4) and $(\epsilon \otimes 1)\left(C_{s s}^{s j}\right)=1$ by (6.7.3) , we have $\left[C_{\alpha s}^{i j}\right]_{13}=0$. This is equivalent to $C_{\alpha s}^{i j}=0$.

Proof of (6.7.6): By (6.7.5) $\Delta\left(u_{11}\right)=\sum_{s} C_{s s}^{11} u_{1 s} \otimes u_{s 1}$. Let $p_{s}=C_{s s}^{11} u_{1 s} \otimes u_{s 1}$ and $p=\sum_{s} p_{s}$. Thus each $p_{s}$ is homogeneous in $H_{1 s} \otimes H_{s 1}$ and so $p_{s}$ commutes with $p_{s^{\prime}}$ for all $s, s^{\prime}$ by Lemma 6.2. Suppose that $p_{s} \neq 0$ for some $s \neq 1$, and fix $m \geq 1$ such that the image of $p_{s}$ is non-zero in $B:=H /\left(z^{m}\right) \otimes H /\left(z^{m}\right)$, using Lemma 6.7(a). To keep the notation within reasonable bounds we shall use the same symbol in what follows for the images of elements in $B$, as for the elements themselves in $H \otimes H$. Since the image of $C_{11}^{11}$ in $H /\langle z\rangle \otimes H /\langle z\rangle=H_{t w} \otimes H_{t w}$ is $1 \otimes 1$ by Proposition (6.6) (d), $C_{11}^{11}$ is invertible in $B$. On the other hand, for all $s \neq 1$, the image of $C_{s s}^{11}$ in $H_{t w} \otimes H_{t w}$ is 0 by Proposition 6.6(d), so the image in $B$ of $C_{s s}^{11}$ is in the Jacobson radical $J:=\operatorname{Jac}(B)$ for all $s>1$. Let $w$ be the maximum integer such that $C_{s s}^{11} \in J^{w}$ for all $s \neq 1$. Fix $i, 1 \leq i \leq n-1$. Because $u_{11}^{n} \in H_{0}$, $p^{n}=\Delta\left(u_{11}^{n}\right) \in H_{0} \otimes H_{0}$. Considering $H \otimes H$ as a $\left(\mathbb{Z}_{n}\right)^{\times 4}$-graded algebra, the degree $(0, i, i, 0)$ part of $p^{n}$ is

$$
n p_{1}^{n-1} p_{i+1}+f_{i}\left(p_{s}\right)
$$

where $f_{i}$ is a polynomial in $\left\{p_{s}: 0 \leq s \leq n-1\right\}$ whose total degree in $p_{0}, p_{2}, \ldots, p_{n-1}$ is at least two. But $p^{n} \in H_{0} \otimes H_{0}$, and so $p^{n}$ is homogeneous of degree $(0,0,0,0)$. It follows that $n p_{1}^{n-1} p_{i+1}+f_{i}\left(p_{s}\right)=0$ for all $i=1, \ldots, n-1$. Therefore

$$
n p_{1}^{n-1} p_{i+1}=-f_{i} \in J^{2 w}
$$

that is,

$$
p_{i+1}=\left(n p_{1}^{n-1}\right)^{-1}\left(-f_{i}\right) \in J^{2 w},
$$

for all $i \neq 0$. This is a contradiction to the definition of $w$. Therefore $p_{s}=0$ for all $s \neq 0$. In other words, $C_{s s}^{11}=0$ for all $s \neq 1$.

Now we are going to prove (a) and (b).

(a) Recall that $\epsilon\left(u_{11}\right)=1$ and $H_{0}$ is the center of $H$. By (6.7.5) and (6.7.6),

$1=\epsilon\left(u_{11}\right)=m_{H} \circ(S \otimes 1) \circ \Delta\left(u_{11}\right)=m_{H}\left[(S \otimes 1)\left(C_{11}^{11}\right)\left(S\left(u_{11}\right) \otimes u_{11}\right)\right]=f S\left(u_{11}\right) u_{11}$ where $f=m_{H} \circ(S \otimes 1)\left(C_{11}^{11}\right)$. Hence $u_{11}$ is invertible.

Since $u_{11}$ is invertible, so is $\Delta\left(u_{11}\right)$, and thus $C_{11}^{11}$ is invertible. Since $C_{11}^{11} \in$ $H_{0} \otimes H_{0}$, and $H_{0}=k[x]$ or $H_{0}=k\left[x^{ \pm 1}\right]$, there is a non-zero scalar $a$ such that $C_{11}^{11}=a$ in the first case or $a x^{i} \otimes x^{j}$ in the second case. It follows from

$$
(\epsilon \otimes 1) \Delta\left(u_{11}\right)=u_{11}=(1 \otimes \epsilon) \Delta\left(u_{11}\right)
$$

that $a=1$ in both cases and $i=j=0$ in the second case. Thus $C_{11}^{11}=1$. The rest of (a) is clear.

(b) We claim first that

$$
\Delta\left(u_{01}\right)=C_{00}^{01} u_{00} \otimes u_{01}+C_{11}^{01} u_{01} \otimes u_{11} .
$$

If $n=2$, 6.7.7) follows from (6.7.5). Suppose now that $n>2$, and let $i \neq j$. Set $s=t \neq j$ and $\alpha=\beta=j$ in (6.7.2) to see that

$$
\left[(\Delta \otimes 1) C_{s s}^{i j}\right]\left[C_{j j}^{i s} \otimes 1\right]=\left[(1 \otimes \Delta) C_{j j}^{i j}\right]\left[1 \otimes C_{s s}^{j j}\right],
$$

and this is zero because $C_{s s}^{j j}=0$ by (a). Since $H_{0} \otimes H_{0} \otimes H_{0}$ is a domain, either $C_{s s}^{i j}=0$ or $C_{j j}^{i s}=0$. However, by Proposition 6.6(e) and (6.6.4), $C_{s s}^{i j} \neq 0$ for all 
$i<s<j$. Therefore $C_{j j}^{i s}=0$ for all $i<s<j$. In particular, $C_{j j}^{01}=0$ for all $j>1$, so that (6.7.7) is proved.

Next we claim that

$$
C_{00}^{01}=C_{11}^{01}=1 \otimes 1 .
$$

Comparing $(\Delta \otimes 1) \Delta\left(u_{01}\right)$ with $(1 \otimes \Delta) \Delta\left(u_{01}\right)$ we obtain the following three equations:

$$
\begin{aligned}
(\Delta \otimes 1) C_{00}^{01} & =\left[(1 \otimes \Delta) C_{00}^{01}\right]\left[1 \otimes C_{00}^{01}\right] ; \\
{\left[(\Delta \otimes 1) C_{11}^{01}\right]\left[C_{00}^{01} \otimes 1\right] } & =\left[(1 \otimes \Delta) C_{00}^{01}\right]\left[1 \otimes C_{11}^{01}\right] ; \\
{\left[(\Delta \otimes 1) C_{11}^{01}\right]\left[C_{11}^{01} \otimes 1\right] } & =(1 \otimes \Delta) C_{11}^{01} .
\end{aligned}
$$

Recall once again the dichotomy of (6.2.3): $H_{0}$ is a central subHopf algebra, either $H_{0}=k[x]$ or $H_{0}=k\left[x^{ \pm 1}\right]$. For $f \in H_{0} \otimes H_{0}$ or $f \in H_{0} \otimes H_{0} \otimes H_{0}$, write $d(f)$ and $\partial(f)$ for the degree of $f$ with respect to $x \otimes 1$ and $1 \otimes x$ respectively in the first case, and with respect to $x \otimes 1 \otimes 1$ and $1 \otimes 1 \otimes x$ respectively in the second case. Write $C_{00}^{01}=\sum_{i, j} a_{i j} x^{i} \otimes x^{j}$ where $a_{00}=c_{11}^{01}=1$.

Case 1: Primitive case: Suppose that $H_{0}=k[x]$, with $x$ primitive. Now (6.7.9) becomes

$$
\sum a_{i j}(x \otimes 1+1 \otimes x)^{i} \otimes x^{j}=\left[\sum a_{i j} x^{i} \otimes(x \otimes 1+1 \otimes x)^{j}\right]\left[\sum a_{i j} 1 \otimes x^{i} \otimes x^{j}\right] .
$$

It follows that $\partial\left(C_{00}^{01}\right)=\partial\left(C_{00}^{01}\right)+\partial\left(C_{00}^{01}\right)$, so that $\partial\left(C_{00}^{01}\right)=0$ and $C_{00}^{01}=\sum_{i} a_{i} x^{i} \otimes 1$. In this case, writing $h:=\sum_{i} a_{i} x^{i}$, 6.7.9) simplifies to

$$
\Delta(h)=(h \otimes 1)(1 \otimes h)=h \otimes h .
$$

But the only group-like element in $k[x]$ is 1 , so that $C_{00}^{01}=1$. A similar argument shows that $C_{11}^{01}=1$, and so (6.7.8) is true in the case $H_{0}=k[x]$.

Case 2: Group-like case: Suppose that $H_{0}=k\left[x^{ \pm 1}\right]$, with $x$ group-like. Since we can switch $x$ and $x^{-1}$ we may assume that $\partial\left(C_{00}^{01}\right) \geq 0$. In this case (6.7.9) becomes

$$
\sum a_{i j}(x \otimes x)^{i} \otimes x^{j}=\left[\sum a_{i j} x^{i} \otimes(x \otimes x)^{j}\right]\left[\sum a_{i j} 1 \otimes x^{i} \otimes x^{j}\right] .
$$

Again $\partial(f)=2 \partial(f)$ and hence $\partial(f)=0$. This says that there is no positive power of $x$ in the second component. Replacing $x$ with $x^{-1}$ we deduce that $f=\sum_{i} a_{i} x^{i} \otimes 1$ and, as in the primitive case, $h:=\sum_{i} a_{i} x^{i}$ is group-like. Therefore $h=x^{i}$ and $C_{00}^{01}=x^{i} \otimes 1$. Replacing $u_{01}$ by $u_{01} x^{-i}$ we have $C_{00}^{01}=1$. A similar argument involving (6.7.11) implies that $C_{11}^{01}=x^{s} \otimes 1$. Then (6.7.10) forces $s=0$ and the proof of (6.7.8) is complete in this case also.

Theorem. Suppose $(H, \pi)$ satisfies Hypotheses 2.5 and (6.1.1). Retain all the notations introduced so far in $\S 6$ for $H$; so, in particular, $H$ is a free $H_{0}$-module with basis of $G_{\pi}^{l} \times G_{\pi}^{r}$-eigenvectors $\left\{u_{i j}: 0 \leq i, j \leq n-1\right\}$, and after Proposition 6.7 $u_{00}=1, u_{i i}=u_{11}^{i}$ is group-like for $i=1, \ldots, n-1$, and $u_{01}$ is $\left(u_{11}, 1\right)$-primitive. Then, after multiplying as necessary by units of $H_{0}$, we can assume that $u_{i j}=$ $u_{11}^{i} u_{01}^{j-i}$ for all $i<j$, and $u_{i j}=u_{11}^{i} u_{01}^{n+j-i}$ for $i>j$. In particular, therefore,

$$
\Delta\left(u_{0 j}\right)=\sum_{s}\left(\begin{array}{l}
j \\
s
\end{array}\right)_{\xi^{t}} u_{0 s} \otimes u_{s j}
$$

and $C_{s s}^{i j}=c_{s s}^{i j} 1 \otimes 1$ for all $i, j, s$. 
Proof. Define $\bar{u}_{i j}:=u_{11}^{i} u_{01}^{j-i}$ for $i \leq j$ and $\bar{u}_{i j}:=u_{11}^{i} u_{01}^{n+j-i}$ for $i>j$. Thus, by Proposition 6.7.

$$
\Delta\left(\bar{u}_{0 j}\right)=\sum_{s}\left(\begin{array}{l}
j \\
s
\end{array}\right)_{\xi^{t}} \bar{u}_{0 s} \otimes \bar{u}_{s j} .
$$

We show first that there are units $f_{j} \in H_{0}$ such that $\bar{u}_{0 j}=u_{0 j} f_{j}$ for $j=$ $0, \ldots, n-1$. Since $\bar{u}_{0 j} \in H_{0 j}$ there are certainly $f_{j} \in H_{0}$ such that these equations hold. Then

$$
\Delta\left(\bar{u}_{0 j}\right)=\Delta\left(u_{0 j} f_{j}\right)=\left(\sum_{s} C_{s s}^{0 j} u_{0 s} \otimes u_{s j}\right) \Delta\left(f_{j}\right) .
$$

As in the proof of (6.7.6), these expressions are $\mathbb{Z}_{n}^{4}$-graded. Equating the degree $(0,0,0, j)$ parts of the two expressions (6.7.12) and (6.7.13) for $\Delta\left(\bar{u}_{0 j}\right)$,

$$
1 \otimes f_{j}=C_{00}^{0 j} \Delta\left(f_{j}\right) \in H_{0} \otimes H_{0} .
$$

Comparing degrees $\partial(-)$ in $1 \otimes x$ in (6.7.14) gives

$$
\partial\left(1 \otimes f_{j}\right)=\partial\left(C_{00}^{0 j}\right)+\partial\left(1 \otimes f_{j}\right),
$$

since $\partial\left(1 \otimes f_{j}\right)=\partial\left(\Delta\left(f_{j}\right)\right)$ whether $H_{0}$ is $k[x]$ or $k\left[x^{ \pm 1}\right]$. Therefore $C_{00}^{0 j}=h \otimes 1$ for some $h \in H_{0}$, which is non-zero since $(\epsilon \otimes \epsilon)\left(C_{00}^{0 j}\right)=1 \otimes 1$ by (6.6.3). Substituting this in (6.7.14) and applying $1 \otimes \epsilon$, we deduce that $\epsilon\left(f_{j}\right) \neq 0$ and then that $f_{j}$ is a unit of $H_{0}$.

Thus $f_{j}$ is either $\lambda_{j}$ or $\lambda_{j} x^{l_{j}}$, where $\lambda_{j} \in k \backslash\{0\}$ and $l_{j} \in \mathbb{Z}$, depending on whether $H_{0}$ is $k[x]$ or $k\left[x^{ \pm 1}\right]$. For each $j=0, \ldots, n-1$, consider the image of the equation

$$
\bar{u}_{0 j}=u_{01}^{j}=u_{0 j} f_{j}
$$

in $H_{t w}$, where it yields

$$
v_{01}^{j}=v_{0 j} \varepsilon\left(f_{j}\right)=v_{01}^{j} \varepsilon\left(f_{j}\right),
$$

in view of the final statement in Theorem (6.6). Hence

$$
\varepsilon\left(f_{j}\right)=1,
$$

so that $\lambda_{j}=1$. Second, in the case where $H_{0}=k\left[x^{ \pm 1}\right]$, we can now replace $u_{0 j}=u_{01}^{j} x^{-l_{j}}$ by $u_{0 j} x^{l_{j}}=u_{01}^{j}$, as required.

Now let $j \geq i \geq 1$, and consider $u_{11} u_{01}^{j-i}$; since $H_{i j}=u_{i j} H_{0}$, we can write

$$
u_{11}^{i} u_{01}^{j-i}=u_{i j} f_{i j}
$$

for some $f_{i j} \in H_{0}$. Thus

$$
H_{0(j-i)}=u_{01}^{j-i} H_{0}=u_{11}^{-i} u_{i j} f_{i j} H_{0},
$$

so that $f_{i j}$ must be a unit of $H_{0}$. Therefore $u_{11}^{i} u_{01}^{j-i} H_{0}=H_{i j}$, so we can replace our initial choice of $u_{i j}$ with

$$
u_{i j}:=u_{11}^{i} u_{01}^{j-i}
$$

as claimed. A similar calculation applies when $i>j$. 


\subsection{Primitive case.}

Proposition. Assume $(H, \pi)$ satisfies Hypotheses 2.5 and (6.1.1). Suppose that $H$ is primitive - that is, the Hopf subalgebra $H_{0}=Z(H)$ is a polynomial algebra $k[x]$. Then $H$ is isomorphic as a Hopf algebra to the Taft algebra $H(n, t, \xi)$ of Example 3.3 with $\operatorname{gcd}(t, n)=1$.

Proof. By Proposition 6.7 $g:=u_{11}$ is group-like and $y:=u_{01}$ is $(g, 1)$-primitive. Since the only group-like in $H_{0}$ is $1, g^{n}=1$. By Lemma (6.2) and the quantum binomial theorem, $y^{n}$ is primitive. Up to a nonzero scalar the only primitive element in $H_{0}$ is $x$, so we may assume that $y^{n}=x$. It then follows that there is a Hopf algebra homomorphism $\phi: H(n, t, \xi) \rightarrow H$. By Theorem 6.7 $H$ is generated by $g$ and $y$, both in the image of $\phi$, and hence $\phi$ is surjective. Since both $H(n, t, \xi)$ and $H$ are prime of GK-dimension $1, \phi$ is an isomorphism.

\subsection{Group-like case.}

Proposition. Assume that $(H, \pi)$ satisfies Hypotheses 2.5 and (6.1.1). Suppose that $H$ is group-like - that is, the Hopf subalgebra $H_{0}=Z(H)$ is a Laurent polynomial algebra $k\left[x^{ \pm 1}\right]$. Then, for appropriate $\xi$ and $w, H$ is isomorphic as a Hopf algebra to one of the algebras $B(n, w, \xi)$ of Example 3.4.

Proof. By Proposition 6.7 $g:=u_{11}$ is group-like and $y:=u_{01}$ is $(g, 1)$-primitive, and these elements together with $x$ generate $H$ as a $k$-algebra by Theorem 6.7. By the $C_{n} \times C_{n}$-grading of $H, g^{n}$ is a group-like element in $H_{0}=H_{00}$, and so there is a $w \geq 0$ such that $g^{n}=x^{w}$, (noting that we can replace $x$ by $x^{-1}$ if $w$ is negative).

Suppose for a contradiction that $w=0$. Then $g^{n}=1$ and hence $y^{n}$ is a primitive element as in Proposition 6.8. But $H_{0}$ has no primitive elements, so we have a contradiction. Therefore we can assume that $w>0$.

By Lemma 6.2 $y g=\zeta^{a} g y$ where $\operatorname{gcd}(a, n)=1$. This implies that $y^{n}$ is a $\left(g^{n}, 1\right)$-primitive element in $H_{0}$. Therefore, after dividing if necessary by a non-zero scalar,

$$
y^{n}=1-g^{n} .
$$

Therefore, in view of Theorem 3.4(c), there is a Hopf algebra homomorphism $\phi$ : $B\left(n, \xi, w, i_{0}\right) \rightarrow H$ given by $\phi: x \rightarrow x, y \rightarrow y, g \rightarrow g$ where $\xi=\zeta^{a}$. Since $H$ is generated by $x^{ \pm 1}, g, y, \phi$ is surjective. However, both $B$ and $H$ are prime of GK-dimension 1 , so $\phi$ is an isomorphism.

The Main theorem 0.5 follows from Theorem 4.1 and Propositions 6.8 and 6.9 The statement in the abstract is an immediate consequence of the Main Theorem.

Corollary. Let $k$ be an algebraically closed field of characteristic zero. Let $H$ be an affine prime regular Hopf algebra of Gelfand-Kirillov dimension one. If the PIdegree of $H$ is 1 or prime, then $H$ is isomorphic to one of the examples listed in 乌3.

Proof. Since $n:=i o(H)$ is equal to the PI-degree of $H, n$ is either 1 or prime. Since $i m(H)$ is a divisor of $i o(H), i m(H)$ must be either 1 or $n$. The result follows from the Main Theorem. 


\section{Questions And COnjectures}

7.1. The most obvious question arising from this paper, one which we hope to address in the future, is:

Question. Is Theorem 0.5 valid without the hypothesis that $i m(H)$ is 1 or $i o(H)$ ?

7.2. The hypotheses - prime versus semiprime. Suppose $H$ is a semiprime regular Hopf algebra of GK-dimension 1 over an algebraically closed field $k$ of characteristic 0. By [BG1, Theorem A] $H$ is a finite direct sum of prime algebras, each of them having GK-dimension 1 because of the Cohen-Macaulay property. It thus seems reasonable to ask:

Question. What are the Hopf algebras $H$ which satisfy $(\mathbf{H})$, but with prime weakened to semiprime?

Of course, it may be easier to tackle this question first in the case where $\mathrm{im}(H)$ is 1 or $i o(H)$.

Since $H$ is a direct sum of prime rings, there is exactly one minimal prime ideal of $H$ - let's call it $P$ - contained in ker $\epsilon$. It is shown in [LWZ, Theorem 6.5 and Lemma 5.5] that $\bar{H}:=H / P$ is a Hopf algebra, and indeed satisfies all the hypotheses $(\mathbf{H})$. Moreover $i o(H)=i o(\bar{H})$ and $i m(H)=i m(\bar{H})$. It's conjectured in [WZ, remark 6.6] that there is a short exact sequence of Hopf algebras

$$
0 \longrightarrow K \longrightarrow H \longrightarrow \bar{H} \longrightarrow 0 \text {. }
$$

Here, $K=H^{c o \bar{H}}$, the coinvariant subalgebra, and $K$ is conjecturally a normal finite dimensional Hopf subalgebra of $H$; if this is true, then it's easy to see that $K$ must be semisimple artinian.

7.3. The hypotheses of the main theorem. (i) The characteristic of $k$ : The assumption that $k$ has characteristic 0 is needed to ensure that $i o(H)$ is a unit. This hypothesis is not a consequence of the others in $(\mathbf{H})$, and without it the theorem is false, as is shown by the example in Remarks (2.3) $\mathrm{B}(\mathrm{a})$. We can thus ask:

Question. What are the noetherian prime affine Hopf $k$-algebras $H$ over an algebraically closed field $k$, with $\mathrm{GK} \operatorname{dim} H=\operatorname{gldim} H=1$ ?

(ii) The algebraic closure of $k$ : When $k$ is not algebraically closed there are in general other 1-dimensional commutative Hopf $k$-domains, besides $k[x]$ and $k\left[x^{ \pm 1}\right]$, [WZ, 8.3]. Therefore we ask:

Question. How should the main theorem be amended if $k$ is not algebraically closed?

(iii) Regularity of $H$ : The non-regular generalised Liu algebras found in (3.4) demonstrate that the remaining hypotheses in $(\mathbf{H})$ do not imply that $H$ has finite global dimension. Here is another example.

Example. Let $n, p_{0}, p_{1}, \cdots, p_{2}$ be positive integers and $q \in k^{\times}$with the following properties:

(a) $s \geq 2$ and $1<p_{1}<p_{2}<\cdots<p_{s}$;

(b) $p_{0} \mid n$ and $p_{0}, p_{1}, \cdots, p_{s}$ are pairwise relatively prime;

(c) $q$ is a primitive $\ell$-th root of unity, where $\ell=\left(n / p_{0}\right) p_{1} p_{2} \cdots p_{s}$. 
Set $m_{i}=p_{i}^{-1} \prod_{j=1}^{s} p_{j}$ for $i=1, \cdots, s$. Let $A$ be the subalgebra of $k[y]$ generated by $y_{i}:=y^{m_{i}}$ for $i=1, \cdots, s$. The $k$-algebra automorphism of $k[y]$ sending $y \mapsto q y$ restricts to an algebra automorphism of $A$. There is a unique Hopf algebra structure on the skew Laurent polynomial ring $B=A\left[x^{ \pm 1} ; \sigma\right]$ such that $x$ is group-like and the $y_{i}$ are skew primitive, with

$$
\Delta\left(y_{i}\right)=y_{i} \otimes 1+x^{m_{i} n} \otimes y_{i}
$$

for $i=1, \cdots, s$. It was checked in [GZ, Construction 1.2] that this is a Hopf PI domain of GK-dimension two, and is denoted by $B\left(n, p_{0}, p_{1}, \cdots, p_{s}, q\right)$.

The Hopf algebra $B$ has infinite global dimension, therefore provides a negative answer to [Bro, Question K] (see [GZ, Remark 1.6]).

Now let $H$ be the quotient algebra $B /\left(x^{\ell}-1\right)$ where $\ell=\left(n / p_{0}\right) p_{1} p_{2} \cdots p_{s}$. Since group-like elements $x^{ \pm \ell}$ generate a central Hopf subalgebra of $B,\left(x^{\ell}-1\right)$ is a Hopf ideal and hence $H$ is a quotient Hopf algebra of $B$.

We claim that $H$ is prime of Gelfand-Kirillov dimension one, but not regular. As an algebra $H$ is a skew group $\operatorname{ring} A * \mathbb{Z} /(\ell)$, whence is semiprime by $\mathrm{Mo}$, Proposition 7.4.8(1)]. Since $\ell$ is the order of $q$, the center of $H$ is $k\left[y^{\ell}\right]$ which is a domain. Hence $H$ is prime. Since $A$ is not regular and $A * Z /(\ell)$ is strongly $\mathbb{Z} /(\ell)$-graded, $A * \mathbb{Z} /(\ell)$ is not regular. Finally $\operatorname{GKdim} H=\operatorname{GKdim} A=1$.

Up to date, we do not know other examples of non-regular prime Hopf algebras of GK-dimension one. Hence we should ask:

Question. What other Hopf algebras have to be included if the regularity hypothesis is dropped from the main theorem?

7.4. GK-dimension greater than one. Beyond GK-dimension one, there is no doubt that many classes of examples remain to be discovered. Of course not every noetherian Hopf algebra of GK-dimension 2 satisfies a polynomial identity: consider the enveloping algebra of the 2-dimensional non-abelian Lie algebra, over a field of characteristic 0 . A few new examples of noetherian Hopf algebras are given in GZ]. However, simply because we don't know many examples, we ask:

Question. Does every noetherian Hopf $k$-algebra of GK-dimension 2 satisfy a polynomial identity if $k$ has positive characteristic?

In arbitrary characteristic, one could start by considering the Hopf algebra case of Small's famous question

Question. Is every noetherian prime Hopf $k$-algebra of GK-dimension 2 either PI or primitive?

Simply for completeness and to emphasis the fact that much remains to be understood about the classical components, we restate the question from Remark (2.3) $\mathrm{B}(\mathrm{b})$ :

Question. If $A$ is an AS-Gorenstein noetherian Hopf algebra with a bijective antipode, is the center of $A$ contained in its classical component?

\section{ACKNOWLEDGMENTS}

Both authors are supported in part by Leverhulme Research Interchange Grant F/00158/X (UK). J.J. Zhang is supported by a the US National Science Foundation. We thank S. Gelaki for helpful advice. 


\section{REFERENCES}

[Bra] A. Braun, An additivity principle for p.i. rings, J. Algebra 96 (1985), 433441.

[Bro] K. A. Brown, Noetherian Hopf algebras. Turkish J. Math. 31 (2007), suppl., 7-23.

[BG1] K. A. Brown and K. R. Goodearl, Homological aspects of Noetherian PI Hopf algebras and irreducible modules of maximal dimension, J. Algebra 198 (1997), 240-265.

[BG2] K. A. Brown and K. R. Goodearl, Lectures on Algebraic Quantum Groups, Birkhäuser, 2002.

[BZ] K.A. Brown and J.J. Zhang, Dualising complexes and twisted Hochschild (co)homology for noetherian Hopf algebras, J. Algebra, 320 (2008), 1814-1850.

[GL] S. Gelaki and E.S. Letzter, An affine PI Hopf algebra not finite over a normal commutative Hopf subalgebra, Proc. Amer. Math. Soc. 131 (2003), 2673-2679

[GS] K.R. Goodearl and L.W. Small, Krull versus global dimension in Noetherian P.I. rings, Proc. Amer. Math. Soc. 92 (1984), 175-178.

[GW] K.R. Goodearl and R.B. Warfield Jr., "An introduction to noncommutative Noetherian rings", Second edition. London Mathematical Society Student Texts, 61. Cambridge University Press, Cambridge, 2004.

[GZ] K.R. Goodearl and J.J. Zhang, Noetherian Hopf algebra domains of Gelfand-Kirillov dimension two, in preparation.

[Hu] J.E. Humphreys, "Linear algebraic groups", Graduate Texts in Mathematics, No. 21. Springer-Verlag, New York-Heidelberg, 1975.

[Liu] G.-X. Liu, On noetherian affine prime regular Hopf algebras of Gelfand-Kirillov dimension 1, Proc. Amer. Math. Soc. (to appear).

[LL] M.E. Lorenz and M.Lorenz, On crossed products of Hopf algebras, Proc. Amer. Math. Soc. 123 (1995), no. 1, 33-38.

[LWZ] D.-M. Lu, Q.-S. Wu and J.J. Zhang, Homological integral of Hopf algebras, Trans. Amer. Math. Soc. 359 (2007), no. 10, 4945-4975.

[MR] J. C. McConnell and J. C . Robson, Noncommutative Noetherian Rings, Wiley, Chichester, 1987.

[Mo] S. Montgomery, Hopf Algebras and their Actions on Rings, CBMS Regional Conference Series in Mathematics, 82, Providence, RI, 1993.

[Pa] D.S. Passman, The Algebraic Structure of Group Rings, Reprint of the 1977 original. Robert E. Krieger Publishing Co., Inc., Melbourne, FL, 1985.

[Pa2] D.S. Passman, Infinite Crossed Products, Academic Press, San Diego, 1989.

[Sc] H.-J. Schneider, Principal homogeneous spaces for arbitrary Hopf algebras, Israel J. Math. 72 (1990), 167-195.

[Sk] S. Skryabin, New results on the bijectivity of antipode of a Hopf algebra, Journal of Algebra, J. Algebra 306 (2006), no. 2, 622-633.

[SSW] L. W. Small, J.T. Stafford and R. B. Warfield Jr., Affine algebras of Gelfand-Kirillov dimension one are PI, Math. Proc. Cambridge Philos. Soc. 97 (1985), no. 3, 407-414.

[Sw] M. E. Sweedler, Hopf Algebras, Graduate Texts in Mathematics, benjamin, New York, 1969.

[V] P. Vamos, On the minimal prime ideal of the tensor product of two fields, Math. Proc. Cambridge Philos. Soc. 84 (1978), no. 1, 25-35.

[Wa] W.C. Waterhouse, Introduction to Affine Group Schemes, Graduate Texts in Mathematics, 66, Springer, Berlin, 1979.

[We] C.A. Weibel, An Introduction to Homological Algebra, Cambridge Studies in Advanced Mathematics, 38. Cambridge University Press, Cambridge, 1994.

[WZ1] Q.-S. Wu and J.J. Zhang, Noetherian PI Hopf algebras are Gorenstein, Trans. Amer. Math. Soc. 355 (2003), no. 3, 1043-1066.

[WZ2] Q.-S. Wu and J.J. Zhang, Regularity of involutary PI Hopf algebras, J. Algebra 256 (2002),no.2,599-610.

[Za] A. Zaks, Injective dimension of semiprimary rings, J. Algebra, 13, (1969), 73-86.

Brown: Department of Mathematics, University of Glasgow, Glasgow G12 8QW, UK

E-mail address: kab@maths.gla.ac.uk

zhang: Department of Mathematics, Box 354350, University of Washington, Seattle, WASHINGTON 98195, USA

E-mail address: zhang@math. washington.edu 